\title{
THE LOCATION, CLUSTERING, AND PROPAGATION OF MASSIVE STAR FORMATION IN GIANT MOLECULAR CLOUDS
}

\author{
Bram B. Ochsendorf ${ }^{1}$, Margaret Meixner ${ }^{1,2}$, Jérémy Chastenet ${ }^{2,3}$, \\ Alexander G. G. M. Tielens ${ }^{4}$, and Julia Roman-Duval ${ }^{2}$ \\ ${ }^{1}$ Department of Physics and Astronomy, The Johns Hopkins University, 3400 North Charles Street, Baltimore, MD 21218, USA; bochsen1@jhu.edu \\ ${ }^{2}$ Space Telescope Science Institute, 3700 San Martin Drive, Baltimore, MD 21218, USA \\ ${ }^{3}$ Observatoire Astronomique de Strasbourg, Université de Strasbourg, CNRS, UMR 7550, 11 rue de l'Université, F-67000 Strasbourg, France \\ ${ }^{4}$ Leiden Observatory, Leiden University, P.O. Box 9513, NL-2300 RA, The Netherlands \\ Received 2016 July 8; revised 2016 September 9; accepted 2016 September 11; published 2016 November 15
}

\begin{abstract}
Massive stars are key players in the evolution of galaxies, yet their formation pathway remains unclear. In this work, we use data from several galaxy-wide surveys to build an unbiased data set of $\sim 600$ massive young stellar objects, 200 giant molecular clouds (GMCs), and $\sim 100$ young ( $<10 \mathrm{Myr})$ optical stellar clusters (SCs) in the Large Magellanic Cloud. We employ this data to quantitatively study the location and clustering of massive star formation and its relation to the internal structure of GMCs. We reveal that massive stars do not typically form at the highest column densities nor centers of their parent GMCs at the $\sim 6 \mathrm{pc}$ resolution of our observations. Massive star formation clusters over multiple generations and on size scales much smaller than the size of the parent GMC. We find that massive star formation is significantly boosted in clouds near SCs. However, whether a cloud is associated with an SC does not depend on either the cloud's mass or global surface density. These results reveal a connection between different generations of massive stars on timescales up to $10 \mathrm{Myr}$. We compare our work with Galactic studies and discuss our findings in terms of GMC collapse, triggered star formation, and a potential dichotomy between low- and high-mass star formation.
\end{abstract}

Key words: galaxies: ISM - galaxies: star formation - Magellanic Clouds - stars: formation - stars: massive

\section{INTRODUCTION}

Massive stars dominate the structure and energy budget of the interstellar medium of galaxies through intense radiation fields, stellar winds, and supernova explosions. However, the pathway that leads to their formation remains unclear, as the process is notoriously difficult to probe because of large distances, crowding, high levels of obscuration, and short lifetimes. In general, star-formation studies have seen dramatic progress in the past decade, which can largely be attributed to the Spitzer Space Telescope and the Herschel Space Observatory. These missions opened up the mid-to-far-infrared (IR) sky at high resolution, allowing us to peek into star-forming cradles that are deeply embedded within giant molecular clouds (GMCs).

The internal structure of GMCs reveal infrared dark clouds (IRDCs) and filaments (up to tens of parsecs), clumps ( $1 \mathrm{pc})$ and cores $(\sim 0.1 \mathrm{pc})$. It is now largely understood that there is an intimate connection between filaments and the formation of low-mass prestellar cores (André et al. 2010, 2014; Könyves et al. 2010). In contrast, studying high-mass clumps and cores has proven to be difficult despite numerous attempts targeting the earliest stages of massive star formation (Motte et al. 2007; Ragan et al. 2012; Schneider et al. 2012; Tackenberg et al. 2012). Recent large surveys of the Galactic plane yield promising results by detecting candidate massive star forming clumps (e.g., Svoboda et al. 2016). Still, confusion and distance ambiguity will inherently complicate studies of massive star formation in the Galaxy and its connection to larger-scale structures in the interstellar medium, e.g., the parent GMCs. Leaving aside the difficulties in probing Galactic massive star formation, there is no theoretical consensus as to the exact physical process that ultimately leads to a (cluster of) massive stars (Tan et al. 2014, p. 149). In this respect, it has long been debated that low-mass stars and high-mass stars may not form in the same way: whereas low-mass cores and stars may form "spontaneously" through hierarchical fragmentation within GMCs (André et al. 2014, p. 27), the formation of high-mass stars may be "triggered" (Elmegreen 1998) by an external mechanism, though the exact nature and/or importance of triggering has remained controversial (see Dale et al. 2015, and references therein).

In this work, we present a galaxy-wide study of massive star formation and its relation with GMCs in the Large Magellanic Cloud (LMC). The LMC provides us with an excellent opportunity to study the formation of massive stars in a wide range of evolutionary stages, since its face-on orientation minimizes confusion and distance ambiguities, while being close enough to resolve individual clouds and stars $(\sim 50 \mathrm{kpc}$; Pietrzyński et al. 2013). By combining several galaxy-wide surveys, we create a unique view of massive young stellar objects (MYSOs), GMCs, and optical stellar clusters (SCs) in the LMC. The multi-facetted nature and sheer size of the data traces massive star formation as a function of environment and evolutionary state, and the overarching goal of this study is to exploit this unique data set to quantify the location, clustering, and propagation of massive star formation within GMCs. In Section 2, we present the observations. In Section 3, we build our catalog of MYSOs, the completeness of which is tested in Section 3.1. We describe the dust fitting and creation of column density maps and subsequent cloud decomposition in Section 4. The distribution of MYSOs within GMCs and its relation to SCs is presented in Section 5. We compare our results with studies performed in the Galaxy, and discuss our findings in relation to recent numerical and analytical studies of collapsing molecular clouds in Section 6. We conclude in Section 7. 


\section{OBSERVATIONS}

In this work, we make use of the far-IR images from the Herschel Inventory of the Agents of Galaxy Evolution (HERITAGE; Meixner et al. 2013) covering the entire IRemitting part of the LMC at 70, 160, 250, 350, and $500 \mu \mathrm{m}$ at $\sim 7^{\prime \prime}, 12^{\prime \prime}, 18^{\prime \prime}, 25^{\prime \prime}$, and $36^{\prime \prime}$ resolution. In addition, we employ data from the Magellanic Mopra Assessment (MAGMA; Wong et al. 2011) Data Release 3 (T. H. Wong et al. 2016, in preparation), a $45^{\prime \prime}$-resolution targeted study of GMCs ( 200 in total; Section 4) with fluxes greater than $1.2 \times 10^{5} \mathrm{~K} \mathrm{~km} \mathrm{~s}^{-1}$ $\operatorname{arcsec}^{2}$ and a completeness limit of $M \sim 3 \times 10^{4} M_{\odot}$.

\section{CATALOG OF MASSIVE YOUNG STELLAR OBJECTS}

We have compiled a catalog of (highly) probable YSOs by combining the results of galaxy-wide searches of YSO candidates (Whitney et al. 2008; Gruendl \& Chu 2009) using Spitzer's Surveying the Agents of a Galaxy's Evolution (SAGE; Meixner et al. 2006) data and HERITAGE data (Seale et al. 2014). These works produced YSO catalogs through careful selection criteria (e.g., color-magnitude cuts, morphological inspection) tailored to minimize contamination from sources such as planetary nebulae, evolved stars, and background galaxies. A certain level of contamination is still expected, with estimates ranging from 55\% (Whitney et al. 2008), 20\%-30\% (Gruendl \& Chu 2009), and <10\% (Seale et al. 2014). However, these levels mainly apply to the faint end of the YSO distribution, which overlap more with the aforementioned contaminants in color-magnitude space than their luminous (i.e., higher-mass) counterparts. For the MYSOs, which are the focus of this study, we expect contamination of our YSO catalogs to be less important.

From Whitney et al. (2008), we use the "YSO candidate" and "high-probability YSO candidate" lists (989 sources). From Gruendl \& Chu (2009), we restrict ourselves to the "probable" and "definite" class of YSO candidates (1171 sources), and from Seale et al. (2014) we employ the list of 2493 "probable" YSOs. These catalogs inherently have overlapping sources, and thus we throw out duplicates by crossmatching the catalogs by finding the nearest on-sky matches between coordinates. We define a match between the SAGE catalogs as coordinates that are within $\leqslant 2^{\prime \prime}$ from one another, while this threshold is raised to $\leqslant 10^{\prime \prime}$ for cross-matching the SAGE and HERITAGE catalogs because of the coarser resolution of the HERITAGE photometric bands (up to 36" for the $500 \mu \mathrm{m}$ band; Meixner et al. 2013). We end up with a final list of 3524 high-probable YSO candidates for the entire LMC.

We also consider the far-infrared "dust clumps" (DCs) discussed in Seale et al. (2014). These dust clumps differ from a HERITAGE candidate YSO through a lack of a $24 \mu \mathrm{m}$ pointsource detection, commonly thought to be a robust tracer of star formation (Dunham et al. 2014, p. 195). Seale et al. (2014) did not include DCs in their final YSO candidate list because the authors revealed that the photometry of these sources could not distinguish between a highly embedded YSO, or a starless ISM clump illuminated by a moderate external interstellar radiation field. However, DCs brighter than $L \geqslant 10^{3} L_{\odot}$ are more luminous than can be explained by the typical radiation field pervading the LMC (Seale et al. 2014), implying the presence of an embedded heating source. Upon closer inspection, Seale et al. (2014) noted that many of the bright DCs reveal extended or saturated $24 \mu \mathrm{m}$ emission, preventing their detection as a point-source in this band and, consequently, eluded classification as a YSO in the SAGE catalog of Whitney et al. (2008). The photometric extraction method employed by Gruendl \& Chu (2009) did allow for extended objects to enter the catalog. In the remainder of this work, we opted to present the results for the DCs separately from that of the MYSOs; however, many DCs may in fact represent true MYSOs due to a $24 \mu \mathrm{m}$ misclassification.

In order to characterize the sources within our catalog, we attempt to fit all sources with the Robitaille et al. (2006) YSO models (except for the DCs, for which there are no suitable models available). The Robitaille et al. (2006) models $\left(2 \times 10^{5}\right.$ in total) cover a wide range of physical parameters for different stages in the YSO evolutionary path, often divided into Stages 1 (least evolved), 2, and 3 (most evolved); see Robitaille et al. (2006) for a definition of these classes. However, it is important to note that the parameters used to divide YSOs in these stages (such as disk mass, envelope accretion, and mass of the central source) are not constrained from the spectral energy distribution (SED) alone (for a thorough discussion, see Robitaille 2008). The bolometric luminosity of the sources is well constrained by the fits, but given that the source luminosity is expected to evolve during the early stages of star formation (e.g., mass accretion), one cannot simply translate observed luminosity into a (main-sequence) mass. Therefore, for the remainder of this study, we chose to define our completeness limits (Section 3.1) and the subsequent source analysis in terms of mass predicted by the Robitaille et al. (2006) models; however, we caution the reader that the reported masses rely on the accuracy of the integrated evolutionary tracks (Robitaille 2008).

We define a "well-fitted" source by one yielding a reduced chi square of $\chi_{\text {red }}^{2} \leqslant 5$. Although arbitrarily chosen, this "chiby-eye" threshold provides a collection of fits that seem very acceptable. From all 3524 YSO sources, 2558 sources have sufficient photometric constraints to be passed to the Robitaille et al. (2006) SED fitter. From these, 1278 sources have $\chi_{\text {red }}^{2} \leqslant 5$, out of which 691 are above our completeness limit (i.e., $M \geqslant 8 L_{\odot}$; Section 3.1). From these 691 MYSOs, we find that 569 are Stage I, 103 are Stage II, and 19 are Stage III. As discussed above, this classification scheme uses parameters not directly related to the SED, but it does provide a handle on the evolutionary state of the source and thereby its age. For lowmass YSOs $\left(\log \left(L / L_{\odot}\right) \lesssim 0.5, M \sim 0.5 M_{\odot}\right)$, the estimated lifetimes of Stage I and Stage II are $\sim 10^{5}$ and $\sim 10^{6}$ years, respectively (Kenyon et al. 1990; Evans et al. 2009). This indicates that our final MYSO list is biased toward young and embedded sources, which is a natural outcome of the selection criteria of the high probable YSO candidate lists (Whitney et al. 2008; Section 3.1). The quoted timescales overestimate the age of our sample since we are tracing the high-mass objects $\left(M \geqslant 8 M_{\odot}, \log \left(L / L_{\odot}\right) \gtrsim 3.5\right)$. For example, the embedded phase for MYSOs with $\log \left(L / L_{\odot}\right)>5.0$ may only last for $<10^{5}$ years (Mottram et al. 2011). In the remainder of this work, we will assume an age of our MYSO sample of $\sim 10^{5}$ year; though, this number does not directly enter our analysis.

\subsection{Completeness Test}

Completeness of the YSO catalogs has been evaluated through false source extraction tests for both the SAGE 

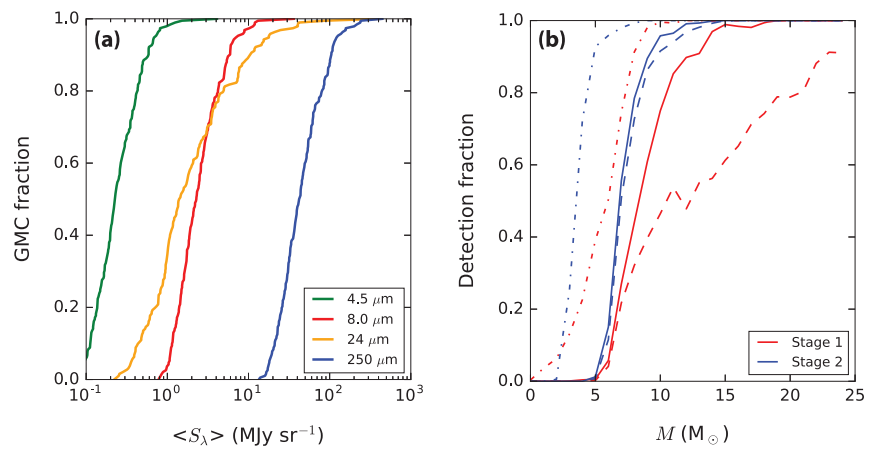

Figure 1. Surface brightness of GMCs and the detection fraction of YSO sources at the distance of the LMC. (a): the average surface brightness of GMCs identified in the LMC (Section 4.2). (b): predicted detection fraction of YSOs as a function of source mass in different of regimes of background level: "LMC average" (dashed-dotted lines), "high background" (solid lines), and the limiting case of "extreme background" (dashed lines). See the text for explanation. Results are shown for Stage 1 and 2 YSOs.

(Gruendl \& Chu 2009) and HERITAGE (Meixner et al. 2013) data. Completeness is mainly limited by the sensitivity of the surveys and the level of the background, which predominantly hampers the detection of faint (i.e., low-mass) YSOs. Luckily, MYSOs are expected to be among the brightest sources detected in the mid-to-far-IR (Whitney et al. 2008; Seale et al. 2014).

Gruendl \& Chu (2009) and Meixner et al. (2013) provide completeness limits as a function of background emission level. For SAGE, we take the completeness limits as given by Gruendl \& Chu (2009) for the highest backgrounds the authors were able to trace, $\sim 10 \mathrm{MJy} \mathrm{sr}^{-1}$, as well as the LMC average, resulting in $1.8(0.1), 3.1(0.2), 3.5(0.2), 5.8(0.4)$, and $24(1.5)$ mJy for high background (LMC average) at 3.6, 4.5, 5.8, 8.0, and $24 \mu \mathrm{m}$, respectively. Similarly, Meixner et al. (2013) provide completeness limits in high background (>2.5-25 $\mathrm{MJy} \mathrm{sr}^{-1}$, depending on the photometric band) and the LMC average of the HERITAGE images, corresponding to 450 (450), 400 (160), 300 (60), 400 (60), and 400 (100) $\mathrm{mJy}$ for high background (LMC average) at 100, 160, 250, 350, and $500 \mu \mathrm{m}$, respectively. This completeness limit was shown by Seale et al. (2014) to be valid up to at least $10^{2} \mathrm{MJy} \mathrm{sr}^{-1}$ for the $250 \mu \mathrm{m}$ band. In this work, we are particularly interested in quantifying the expected detection fraction of YSOs within GMCs. As molecular clouds may lie amid bright dust emission associated with star formation, we will investigate the detection fraction of YSOs in regions of high background. Figure 1(a) shows that the average surface brightness $\left\langle S_{\lambda}\right\rangle$ of the vast majority of GMCs identified in the LMC $(\sim 200$ in total; Section 4.2) lie below the threshold identified for "high background" (10 MJy sr ${ }^{-1}$ for the 4.5, 8.0, and $24 \mu \mathrm{m}$ bands, $10^{2} \mathrm{MJy} \mathrm{sr}^{-1}$ for the $250 \mu \mathrm{m}$ band).

We use the Robitaille et al. (2006) YSO models to translate from flux to mass space: using the models, we predict the observed flux from a YSO at the distance of the LMC, which we compare with the aforementioned completeness limits at a given background level. We consider a YSO to be detected if the predicted flux from the model exceeds that of our completeness limit in at least three photometric bands. We do not include the 2MASS and MIPS $70 \mu \mathrm{m}$ filters in the completeness test since, for these bands, the completeness limits have not been investigated. This also means that we cannot address the completeness for Stage 3 sources, which predominantly emit at optical to near-IR wavelengths (i.e., 2MASS). Figure 1(b) shows that the SAGE/HERITAGE observations should be most sensitive to Stage 2 sources, but the stringent color cuts applied by Whitney et al. (2008) and Gruendl \& Chu (2009) to separate YSOs from foreground and background contaminants renders our census of Stage 2 (and Stage 3) sources incomplete. However, these sources are largely irrelevant to this work since we aim to probe the youngest population of YSOs, i.e., the earliest stages of star formation. Indeed, the "allowed" mid-IR color space encompasses the predicted colors of Stage 1 sources (Whitney et al. 2008): the youngest, most embedded sources that shine brightly in the mid-to-far-IR, presumably as they did not have time to dissipate their surrounding material.

We consider the detection fraction of Stage 1 MYSOs $\left(M \geqslant 8 M_{\odot}\right)$. Figure $1(\mathrm{~b})$ shows that, averaged over the LMC, we recover $\sim 90 \%$ of the Stage 1 MYSOs. Even within regions of high background (which again is not representative of our entire sample; Figure 1(a)), we recover the majority $(>50 \%)$ of Stage 1 MYSOs, a fraction that quickly rises with source mass $M$. Finally, we consider the limiting case of 30 Doradus. At short wavelengths, diffuse background emission from warm dust and/or PAHs can arise in areas that are highly illuminated by nearby massive stars (e.g., clouds near 30 Doradus), especially at 8 and $24 \mu \mathrm{m}$. At far-IR wavelengths, the emission from the diffuse ISM or cold dust can become significant, while the increasing beam size toward longer wavelengths will decrease the contrast of a (point-like) YSO with its surroundings. To estimate our ability to detect MYSOs in the extreme background of the 30 Doradus region, we raise the surface brightness thresholds for the "high-background" regions (see above) by an order of magnitude, assume that the completeness limits follow surface brightness linearly, and re-evaluate our detection fractions. Visual inspection of the SAGE images reveal that even in the 30 Doradus region the background does not exceed $\sim 10 \mathrm{MJy} \mathrm{sr}^{-1}$ at 3.6, 4.5, and $5.8 \mu \mathrm{m}$, and therefore we do not raise the completeness limits for these bands. Figure 1 shows that even within this case of extreme background, we recover the majority of Stage 1 source of $M \gtrsim 10 M_{\odot}$.

The success of recovering MYSOs can be attributed to the large contrast of MYSOs with the ISM at mid-IR wavelengths (see Figure 2). Note that many of the LMC MYSOs may eventually break up in small clusters given our limited resolution; however, it is expected that the source luminosity is dominated by its highest-mass member since $L \propto M^{\alpha}$, with $\alpha>1$ (Tout et al. 1996). We conclude that our catalog of YSOs should be complete for Stage 1 sources of $M \geqslant 8 M_{\odot}$ because they are bright enough to be detected in the SAGE/HERITAGE surveys; though, some sources without a point-source counterpart in the mid-IR may have eluded detection within extreme regions of IR background. The analysis in the remainder of this work is based exclusively on the 569 Stage 1 MYSOs.

\section{GMCS: COLUMN DENSITIES AND SUBSTRUCTURE}

In this work, we are particularly interested in characterizing the distribution of material within GMCs and its relation to the MYSOs. Column density maps can be derived either from the far-IR HERITAGE images (dust-based) or the ${ }^{12} \mathrm{CO}(1-0)$ emission from the MAGMA survey (gas-based). 
TYPE 1

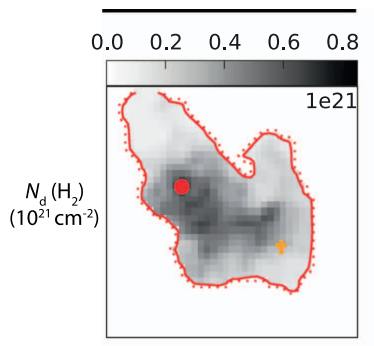

\begin{tabular}{lllll}
0.0 & 0.2 & 0.4 & 0.6 & 0.8 \\
\hline
\end{tabular}

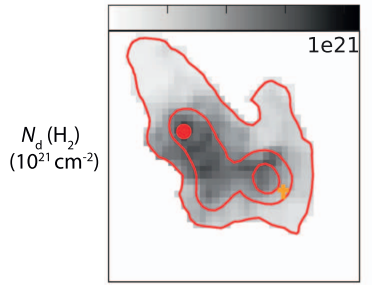

$21.0 \quad 22.5 \quad 24.0 \quad 25.5$
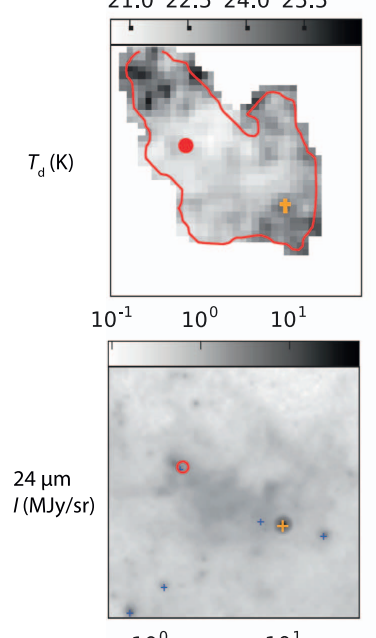

$10^{0}$

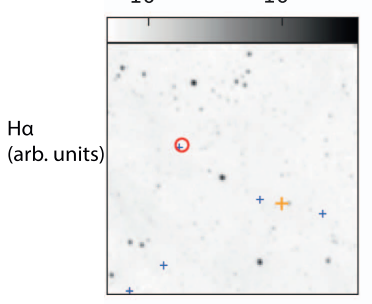

$+\operatorname{MYSO}\left(M>8 M_{\text {sun }}\right)$
TYPE 2
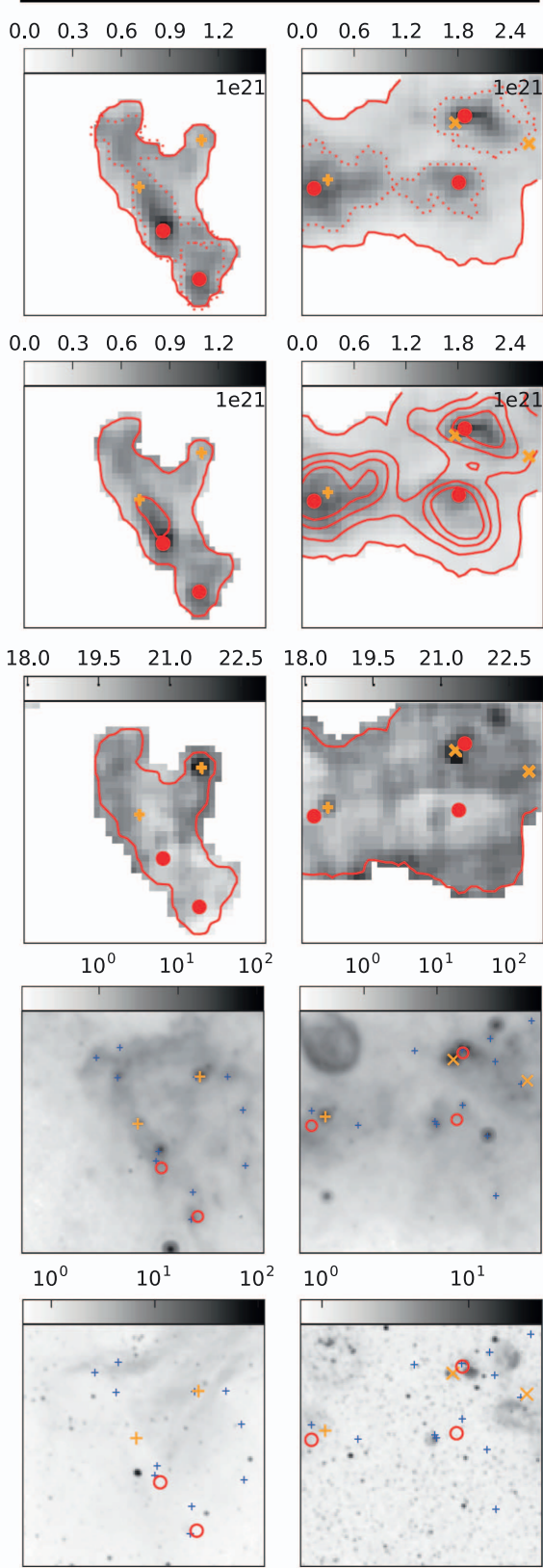

$\begin{array}{lllll}0.0 & 0.6 & 1.2 & 1.8 & 2.4\end{array}$
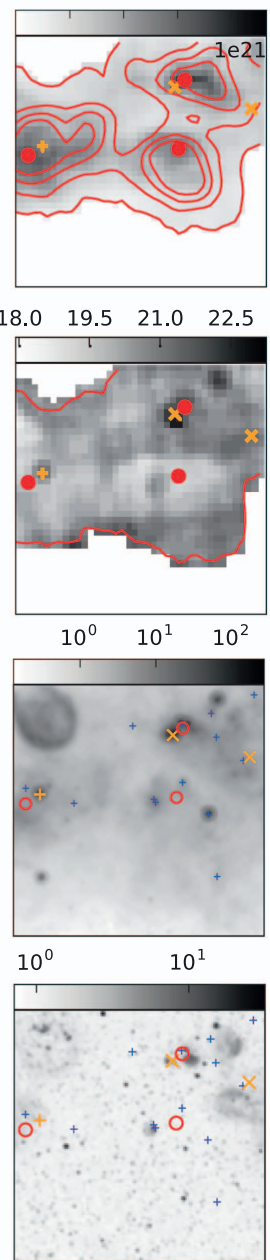

$\times \operatorname{vmYso}\left(M>25 M_{\text {sun }}\right)$

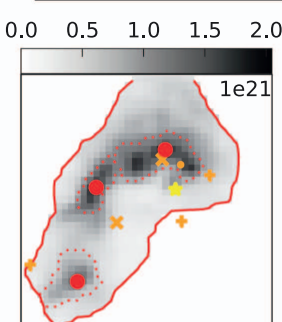

$\begin{array}{lllll}0.0 & 0.5 & 1.0 & 1.5 & 2.0\end{array}$
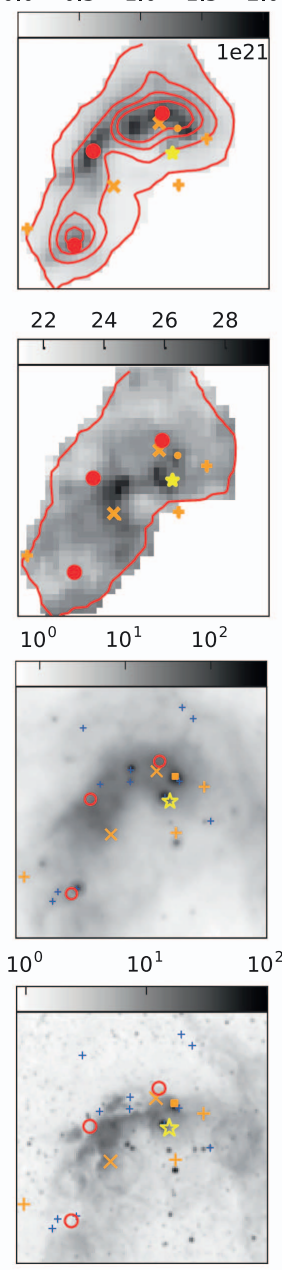

DC $\left(\log \left(L_{\text {sun }} / L\right)>3.5\right)$
TYPE 3
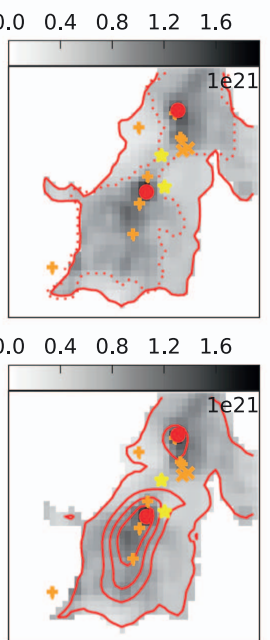

$20.0 \quad 22.5 \quad 25.0 \quad 27.5$
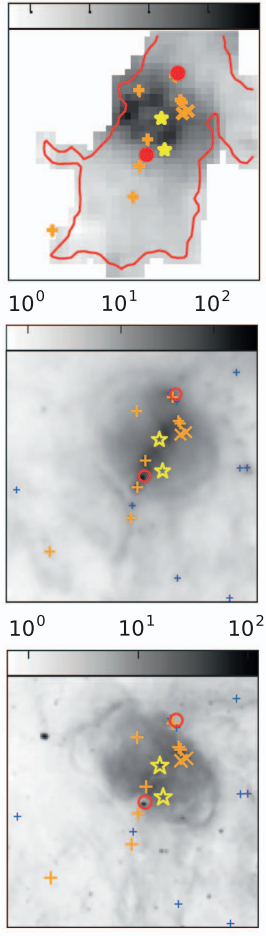

$N_{\mathrm{d}, \max }$
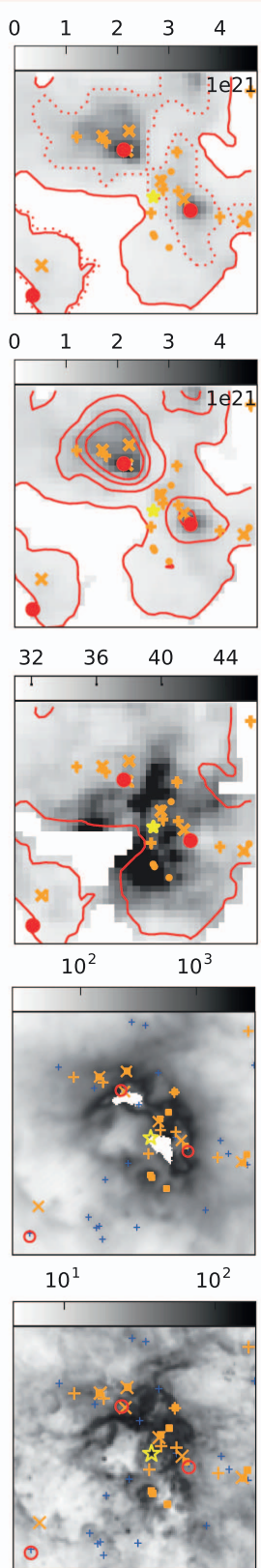

$\star S C$

Figure 2. Internal structure of GMCs and its relation to stellar sources. Examples are shown for Type 1 (GMCs with no associated H II region and/or SC; Kawamura et al. 2009), Type 2 (GMCs with associated H II regions), and Type 3 (GMCs with associated H II regions and optical stellar clusters). Overplotted in all panels: massive young stellar objects (MYSOs; orange plus symbols), very massive young stellar objects (VMYSOs; orange cross symbols), dust clumps (DCs; orange dots), and optical stellar clusters (yellow asterisks; Kawamura et al. 2009). First row: total column density of $\mathrm{H}_{2}$ as revealed by FIR emission. We show the lowest level of the dendrogram structure ("islands"; red solid line), defined as the $3 \sigma$ sensitivity limit of the MAGMA survey (Wong et al. 2011). The highest level of substructure identified by the dendrogram algorithm within the islands are overplotted ("clouds"; dotted red line) as well as the location of its peak value (" $N_{\max }$ "; red dot). Second row: same image as in the top row, but now overlaid with ${ }^{12} \mathrm{CO}(1-0)$ emission contours, offering an independent measure of column density. The gas and dust are found to be in good agreement with each other. Third row: dust temperature maps, overlaid with island footprints and locations of $N_{\max }$. Fourth row: MIPS $24 \mu \mathrm{m}$ intensity maps (see the online version for a high resolution of these figures). In this panel, we have removed the filling of the red dots $\left(N_{\max }\right)$ and yellow asterisks (SCs) to better reveal their background, and we have added sources from the entire catalogue of YSOs (blue plus symbols), which contain sources either below our completeness limit or those that remain uncharacterized (Section 3). Depending on their evolutionary state, MYSOs are seen in sharp contrast with their surroundings at $24 \mu \mathrm{m}$ (Section 3.1), except in a region of extreme background, such as the 30 Doradus region (outermost right panel), part of which is saturated in the $24 \mu \mathrm{m}$ band. Fifth row: MCELS (Smith \& MCELS Team 1998) H $\alpha$ images (uncalibrated), revealing that Stage 1 MYSO do not have an optical counterpart, confirming the embedded nature of these sources. All images span $\sim 100 \times 100 \mathrm{pc}$ at the distance of the LMC.

It is well known that ${ }^{12} \mathrm{CO}(1-0)$ emission alone is an unreliable tracer of mass concentration within GMCs harboring massive star formation, since there are numerous pathways that may affect the ${ }^{12} \mathrm{CO}(1-0)$ emission. The principle advantage of dust over gas in column density estimates is the dynamic range probed; gas tracers are only sensitive to a specific range in volume densities that relate to critical densities, depletion, and opacity effects. In addition, Madden et al. (1997) found 
evidence for hidden molecular hydrogen not traced by $\mathrm{CO}$ in low-metallicity irregular galaxies using the [C II] $158 \mu \mathrm{m}$ line, while Bernard et al. (2008) noted a hidden molecular phase (i.e., "CO-dark gas") through the presence of an FIR excess emission that cannot be explained through $\mathrm{HI}$, and yet does not correlate with $\mathrm{CO}$ emission. This hidden phase may be significant in the low-metallicity environments of the Magellanic clouds (Jameson et al. 2016). Lastly, heating by young massive stars may affect the $\mathrm{CO}$ emissivity per unit mass (Scoville et al. 1987), questioning the validity of a "constant" $X_{\mathrm{CO}}$ factor within regions of massive star formation. We conclude that for the purpose of this study, using the FIR dust emission to trace molecular gas represents a more direct and robust method that avoids the known biases of $\mathrm{CO}$ as a tracer of $\mathrm{H}_{2}$ and, therefore, we proceed our investigation by solely using dust-based column density maps.

\subsection{Far-infrared Column Densities}

We fit the dust far-IR SED on a pixel-to-pixel basis (pixel size $10^{\prime \prime}$ ) assuming optically thin emission using a singletemperature blackbody modified by a power-law emissivity, $I_{\lambda}=\Sigma_{\mathrm{d}} \kappa(\lambda, \beta) B_{\lambda}\left(T_{\mathrm{d}}\right)$. Here, $\Sigma_{\mathrm{d}}$ is the dust surface density, $B_{\lambda}$ is the Planck function, $\kappa_{\lambda}=\left(\kappa_{\text {eff }} / 160^{-\beta}\right) \lambda^{-\beta}$ the emissivity law with $\kappa_{\lambda}$ the dust emissivity at wavelength $\lambda$, $\kappa_{\text {eff }}=28.9 \mathrm{~cm}^{2} \mathrm{~g}^{-1}$ the dust emissivity at reference wavelength $\lambda=160 \mu \mathrm{m}$ (Gordon et al. 2014), and $\beta$ the spectral index. Note that the value for $\kappa_{\text {eff }}$ is larger than the value given in Gordon et al. (2014); the reported value was erroneously tabulated as $\kappa_{\text {eff }} / \pi$. This error did not propagrate into the analysis or results (K. D. Gordon 2016, private communication). We fit the HERITAGE photometric data following the method described in Gordon et al. (2014), and leave $\Sigma_{\mathrm{d}}, T_{\mathrm{d}}$, and $\beta$ as free parameters. The Gordon et al. (2014) fit method reduces the degeneracy between, e.g., $T_{\mathrm{d}}$ and $\beta$ (Dupac et al. 2003; Shetty et al. 2009) by accounting for the correlated errors between the Herschel bands, while using the full likelihood function for each parameter (i.e., the expectation value), as opposed to $\chi^{2}$ minimizations that only use the maximum value of the likelihood. The submilimeter excess, defined as the excess emission seen at submillimeter wavelengths above that expected for dust grains at a single temperature and $\lambda^{-\beta}$ emissivity law (i.e., our adopted model), contributes $27 \%$ to the observed $500 \mu \mathrm{m}$ flux averaged over the entire LMC (Gordon et al. 2014). Based on observed gas-todust ratios, Gordon et al. (2014) argue that the submillimeter excess is more likely to be due to emissivity variations than a second population of very cold dust. For this reason, we have opted to exclude the SPIRE $500 \mu \mathrm{m}$ band in our model fitting: while we sacrifice a data point on the Rayleigh-Jeans part of the SEDs, we avoid contamination by submillimeter excess emission that cannot be captured by our single-temperature model. At the same time, this choice increases the resolution of our dust model maps from $\sim 36^{\prime \prime}$ ( $9 \mathrm{pc}$ ) to $\sim 25^{\prime \prime}$ (6 pc), which constitutes a significant improvement to the cause of our study, since we aim to relate the location of MYSOs to the internal structure of GMCs. From $\Sigma_{\mathrm{d}}$, we convert to molecular hydrogen column density through $N\left(\mathrm{H}_{2}\right)=R \Sigma_{\mathrm{d}} / \mu_{\mathrm{H}} m_{\mathrm{H}}$, where $R$ is the gas-to-dust ratio in the LMC $(\approx 380$; Roman-Duval et al. 2014), $m_{\mathrm{H}}$ is the mass of a hydrogen atom, and we take $\mu_{\mathrm{H}}=2.8$ as mean molecular weight per hydrogen molecule.

\subsection{Deconvolution of GMCs}

We chose to deconvolve the hierarchical structure of the dust column density of GMCs using the dendrogram technique (see Rosolowsky et al. 2008). Dendrograms trace local significant maxima and the way these maxima are connected along isocontours. Compared to other cloud-decomposing algorithms such as CLUMPFIND (Williams et al. 1994), dendrograms have been shown to be more robust against noise and userdefined parameters (Goodman et al. 2009; Pineda et al. 2009). The HERITAGE data suffer from residual striping effects along the PACS/SPIRE scan directions that propagate as fluctuations in our column density maps with a level of $\Delta N\left(\mathrm{H}_{2}\right) \sim 1-4 \times 10^{20} \mathrm{~cm}^{-2}$. To avoid being biased by residual artifacts in our column density maps, we define local maxima $\left(N_{\max }\right)$ as a structure that has a minimum column density contrast of $\Delta N\left(\mathrm{H}_{2}\right)=8 \times 10^{20} \mathrm{~cm}^{-2}$, while containing a minimum number of pixels that exceeds the beam area of the HERITAGE survey (Section 2) by a factor of two. Since we are interested in the properties of GMCs, we limit the deconvolution of the dust-based column density maps to regions of the LMC that exhibit significant $\mathrm{CO}$ emission, which we obtain from MAGMA integrated intensity maps with an rms noise level of $\sigma_{\text {noise }} \sim 0.4 \mathrm{~K} \mathrm{~km} \mathrm{~s}^{-1}$ (Wong et al. 2011). Note that by restricting the dust maps to MAGMA positive detections, we may exclude the more diffuse areas of GMCs projected on the sky, i.e., the "CO-dark" phase (Section 4). However, in this work, we are tracing the formation of massive stars within GMCs, which is very likely to occur in high volume density regimes of GMCs at column depths large enough for $\mathrm{CO}$ to survive ("CO-bright" regions).

We follow the nomenclature of Wong et al. (2011) and Hughes et al. (2013) and refer to the largest contiguous structures of CO emission detected by MAGMA as "islands." The internal column densities of individual islands are subsequently derived using the higher-resolution dust-based column density maps (see Figure 2 for several examples). Note that at higher resolution, the LMC GMCs appear less extended than implied by the CO-based islands, since $N\left(\mathrm{H}_{2}\right) \lesssim 10^{21} \mathrm{~cm}^{-2}$ in parts of the island footprints, which are regions where $\mathrm{CO}$ is expected to be dissociated and not detectable (e.g., Bolatto et al. 2013). Moreover, the dust-based column density maps show large-scale density enhancements, from localized density peaks to filaments of tens of parsecs in size. We define a "cloud" as the highest column density structure within an island as identified by the dendrogram analysis (Figure 2). In the remainder of this paper, "islands" and "clouds" exclusively refer to the products of our dendrogram decomposition.

\section{THE DISTRIBUTION OF MASSIVE STAR FORMATION WITHIN GMCs}

Kawamura et al. (2009) classified GMCs in the LMC as Type 1 (GMCs with no massive star formation), Type 2 (GMCs with associated H II regions), and Type 3 (GMCs with associated $\mathrm{H}$ II regions and optical SCs). This classification was based on GMCs detected in the NANTEN survey (at a resolution of 2!'6; Fukui et al. 2008), not all of which have been observed by MAGMA (at a resolution of 45"; Wong et al. 2011), but a lot of which reveal substructure at higher resolution. To directly compare with the work of Kawamura et al. (2009), we consider all MAGMA islands detected within 
Table 1

Embedded Massive Star Formation in GMCs

\begin{tabular}{lccc}
\hline \hline & Number & $p_{\text {MYSO }}$ & $\left\langle N_{\text {MYSO }}\right\rangle$ \\
\hline Type 1 (island) & 42 & $14 \%$ & 0.2 \\
Type 2 (island) & 93 & $49 \%$ & 1.0 \\
Type 3 (island) & 52 & $75 \%$ & 3.9 \\
Type 1 (cloud) & 72 & $9 \%$ & 0.1 \\
Type 2 (cloud) & 160 & $33 \%$ & 0.6 \\
Type 3 (cloud) & 213 & $42 \%$ & 0.9 \\
\hline
\end{tabular}

Note. Listed are parameters for "islands" and "clouds": total number of islands/clouds found in the dendrogram-based decomposition (Section 4.2), the percentage of islands/clouds with an embedded MYSO, $p_{\text {MYSo }}$; the mean amount of MYSOs per island/cloud, $\left\langle N_{\text {MYSO }}\right\rangle$. The different GMC "Types" stem from the classification of Kawamura et al. (2009).

the footprint of a NANTEN GMC as being of the same "Type." The results are shown in Table 1. We find 42, 93, and 52 Type 1 islands, Type 2 islands, and Type 3 islands, which are further decomposed into 72, 160, and 213 individual clouds. We note that 74 individual structures are not matched. These unmatched structures partially represent structures observed with MAGMA that were not detected with the NANTEN survey, but are mostly small fragments that fall outside of the ellipsoid footprints defined by the NANTEN catalog (Fukui et al. 2008) and are separated from an island at the higher resolution of MAGMA. Indeed, even though appearing large in number, the combined $\mathrm{CO}$ mass incorporated in these 74 fragments is only $4 \%$ of the entire luminous CO mass detected by MAGMA. Given this, we exclude these unmatched structures to our further analysis because we expect they will not affect the conclusions in this paper.

We recover a total of 311 MYSOs within the CO island boundaries (out of 569 total; Section 3), which implies that almost $50 \%$ of MYSOs have not been associated with $\mathrm{CO}$ emission in the MAGMA survey, which was already noted by Wong et al. (2011). This mainly results from the incomplete coverage of the MAGMA survey, but also because the survey is insensitive to clouds below $M \sim 3 \times 10^{4} M_{\odot}$, as well as possible molecular cloud disruption through massive star feedback (see for a discussion Wong et al. 2011).

The average number of MYSOs per island, $\left\langle N_{\text {MYSO }}\right\rangle$, equals 0.2 , 1.0, and 3.9 for Type 1, Type 2, and Type 3 islands, respectively (Table 1). The percentage of Type 1 islands that shows at least one MYSO is 14\% (an example of which is shown in Figure 2). This means that the classification by Kawamura et al. (2009) is largely consistent with our MYSO census, a result that may be surprising given that the authors did not include in their analysis the young, dust enshrouded phase of star formation revealed by the SAGE and HERITAGE surveys. The quantities $p_{\text {MYso }}$ and $\left\langle N_{\text {MYso }}\right\rangle$ increase in Type 2 and Type 3 islands, confirming that these regions are more actively forming massive stars (Kawamura et al. 2009). Note that these numbers shift downward when considering clouds as opposed to islands, which implies that massive star formation occurs in specific parts of islands, rendering the majority of clouds devoid of any (Stage 1) MYSO. Indeed, averaged over the entire galaxy, only $33 \%$ of the LMC clouds ( $48 \%$ when considering islands) show evidence for ongoing massive star formation over the past $\sim 10^{5}$ years (estimated lifetime of Stage 1 MYSOs; Section 3).

\subsection{Location of Massive YSOs in GMCs}

Figure 2 compares GMC column density maps with our YSO catalog. We plot the locations of stellar sources for several subgroups in which we have estimated that our census is complete (Section 3.1). The first group includes the MYSOs of $M \geqslant 8 M_{\odot}$ (main-sequence mass of $\sim \mathrm{B} 2 \mathrm{~V}$ star; Mottram et al. 2011). From the MYSOs, we take the most luminous sources and define a subset of very massive young stellar objects ("VMYSOs") of $M \geqslant 25 M_{\odot}$ (main-sequence mass of $\sim 07.5 \mathrm{~V}$ star). Note that the division of MYSOs and VMYSOs may be equivalent to separating the progenitors of $\mathrm{B}$ and $\mathrm{O}$ stars, respectively. Lastly, we include the "DCs"; given that we have not been able to derive masses for this class (Section 3), we rely on the luminosity of the source and translate this to a main-sequence mass. We include DCs with $\log \left(L / L_{\odot}\right) \geqslant 3.5$, the luminosity equivalent of a $M \geqslant 8 M_{\odot}$ main-sequence star (Mottram et al. 2011). Together, these subsets define a complete tally of the youngest $\left(\sim 10^{5}\right.$ year $)$ sources on their way to becoming massive stars.

Figure 2 shows that the positions of MYSOs, VMYSOs, and DCs do not seem to correlate well with the local column density peaks $N_{\max }$. Upon close inspection, one may argue that MYSOs tend to avoid the highest column densities within GMCs, and are instead often positioned against the outskirts or edges of column density enhancements within clouds/islands. These density enhancements have typical sizes of up to tens of parsecs, similar to infrared dark clouds in the Galaxy (IRDCs; Rathborne et al. 2006). To quantify the relative distribution between MYSOs and $N_{\max }$, we cross-match the locations of our catalog (Section 3) with those of $N_{\max }$ (Section 4). For each MYSO, we find its nearest on-sky $N_{\max }$, after which we plot the column density ratio at the location of the YSO, $N_{\text {YSO }}$, over that of its matched column density peak, i.e., $N_{\mathrm{YsO}} / N_{\max }$. A value of 0.0 of this ratio would mean that the source is located outside of an island (we only count MYSOs located within an island), whereas a value of 1.0 means that the source is located within the pixel containing $N_{\max }$.

Figure 3 shows the results of this analysis, where we further break up the results in Type 1, Type 2, and Type 3 regions. The trend seen in Figure 2 is immediately revealed: there is a clear deficit of sources at high column densities. This holds for both the MYSO as the VMYSO distributions, and is robust against the user-defined inputs of the dendrogram decomposition (Section 4). The DCs are hampered by small number statistics, but this subset does seem to favor higher column densities compared to the MYSO/VMYSO distributions. The gray area in the highest column density bin represents sources that fall within the pixel of $N_{\max }$, and are thus unresolved in this positional analysis. This resolution effect stems from the relative coarse pixel scale of our column density maps $\left(10^{\prime \prime}\right.$ or $2.5 \mathrm{pc}$ at the distance of the LMC), causing all sources found within the pixel of peak column density to collapse in this histogram bin. We expect that, in reality, these sources will form an extended wing of the distribution, possibly declining toward $N_{\text {YSO }} / N_{\max }=1.0$. The positions of the MYSOs are known at a higher accuracy compared to our column density maps, since their detections are matched to shorter wavelength measurements, including Spitzer and 2MASS (Meixner et al. 2013; Seale et al. 2014). The sources found within the pixel of $N_{\max }$ show a smooth distribution with distance as measured from the center of $N_{\max }$ (see below; Figure 4), 

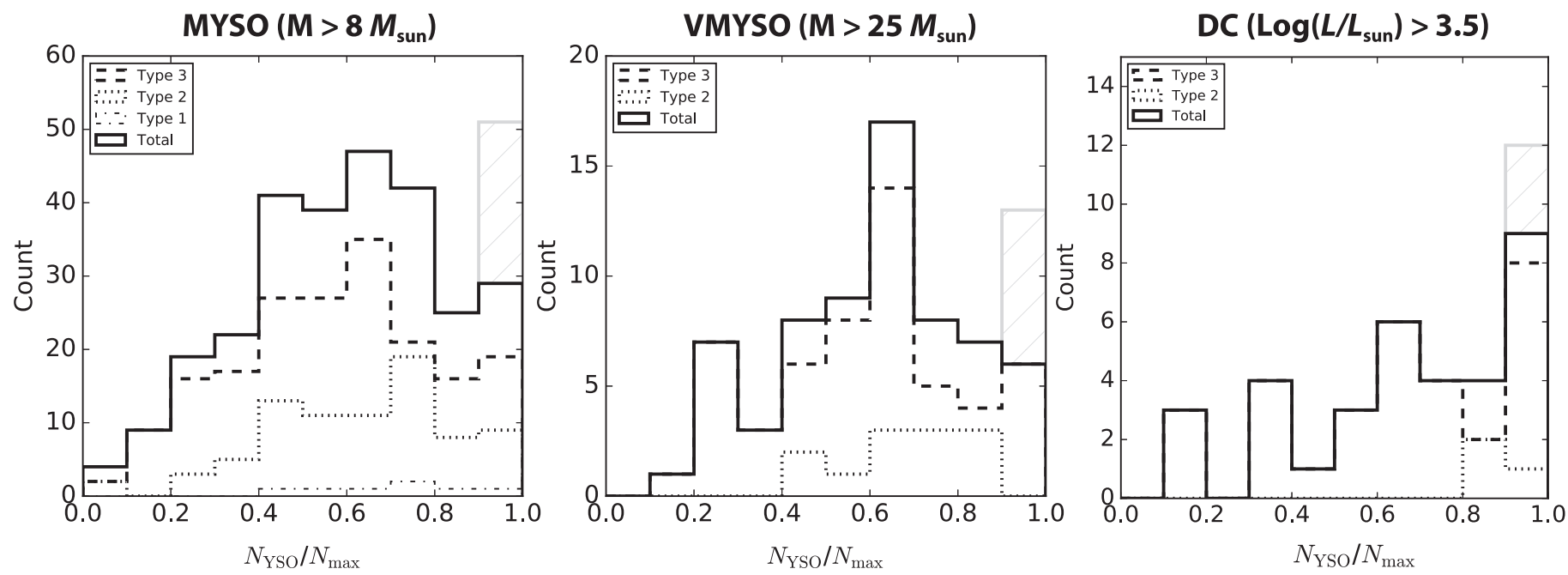

Figure 3. Relative column densities of MYSOs (left panel), VMYSOs (middle panel), and DCs (right panel) with respect to local $\mathrm{H}_{2}$ column density peaks traced by FIR dust emission ( $N_{\max }$; Figure 2). The results are shown for Type 1, Type 2, and Type 3 clouds, as well as the total distribution. The gray hatched area represents sources that fall within the pixel of $N_{\max }$, and are thus unresolved in this positional analysis (see the text).

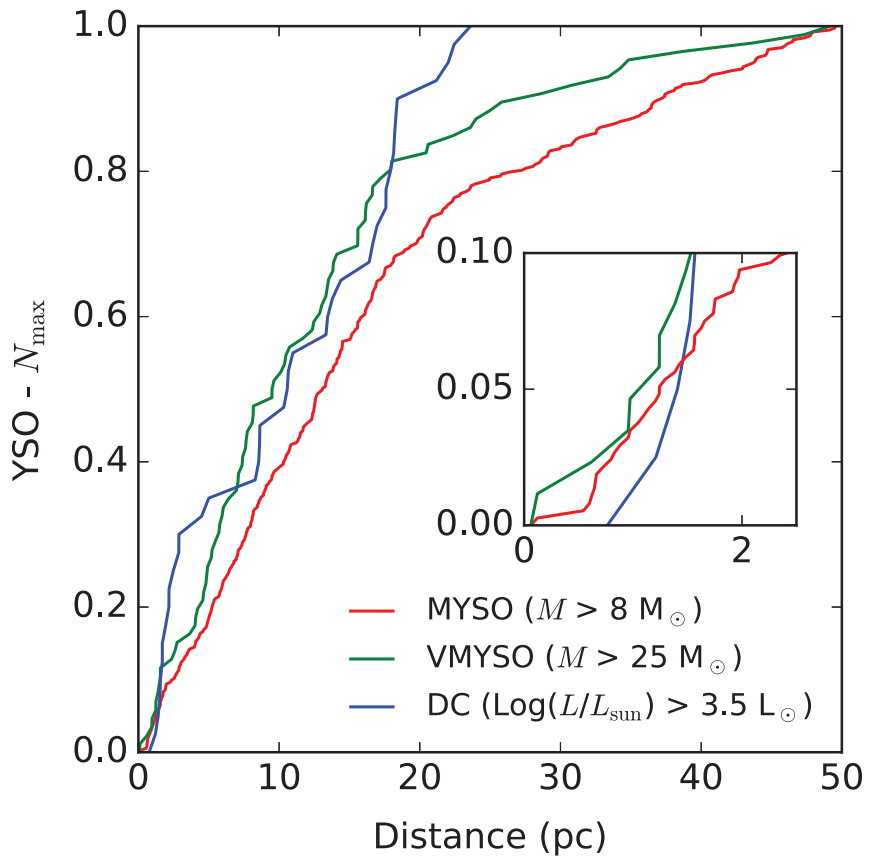

Figure 4. Cumulative distribution of the distance between YSOs and the nearest on-sky column density peaks $\left(N_{\max }\right)$. Plotted are MYSOs (red), a subset of VMYSOs (green), and DCs (Section 3; blue).

revealing that there is structure that is unresolved in our $N_{\text {Yso }} / N_{\text {max }}$ histograms.

As a caveat, we note that the exact shape of the histograms in Figure 3 are biased by projection effects (i.e., the threedimensional distribution of sources with respect to the GMCs), and an "aperture" effect (i.e., the effective area each bin in Figure 3 traces). Whereas the former would only increase the dearth of YSOs toward high column densities if part of the YSOs are found at the position of $N_{\max }$ due to the changing alignment along the line of sight, the latter depends on the internal density distribution of each individual cloud. A sharply peaked density profile of GMCs will cause the higher density bins of Figure 3 to trace only a small part of the cloud in spatial terms. Still in this case, if massive stars would form at the highest column densities of GMCs, we would expect to see a strongly peaked profile skewed toward $N_{\mathrm{YSO}} / N_{\max } \sim 1.0$.

The sample size in Type 1 islands (six in total for the MYSOs) are too small for a statistical analysis, which would have provided insight into the formation of massive stars in presumably the least evolved or youngest GMCs (Kawamura et al. 2009). For Type 2 and Type 3 clouds, we see that the distributions peak at roughly $N_{\mathrm{YSO}} / N_{\max } \sim 0.6-0.65$. Interestingly, in Type 3 islands, the chance of finding a VMYSO in the outskirts of a cloud/island $\left(N_{\mathrm{YSO}} / N_{\max } \sim 0.2\right)$ is similar to that near its peak column density $\left(N_{\mathrm{YsO}} / N_{\max } \sim 1.0\right)$. Even though small in number, we note that DCs appear to favor high column densities.

Figure 4 shows the cumulative distribution of the distance between MYSOs, VMYSOs, and DCs with the nearest column density peak $N_{\text {max }}$. The figure shows that VMYSO and DC objects tend to be located closer to $N_{\max }$ compared to MYSOs. The similarity of the distribution may indicate that DCs represent part of the VMYSO distribution that has been misclassified in our YSO catalog (Section 3). Nonetheless, half the objects within all subsamples are found $\sim 10 \mathrm{pc}$ away from $N_{\max }$. The inset shows the distribution of sources within the pixel of $N_{\max }$ (as measured from the pixel center), showing a structure that is unresolved in our column density maps.

\subsection{Clustering and the Connection between Different Generations of Massive Star Formation}

Given that we have obtained a unique set of MYSOs and GMCs throughout an entire galaxy, we are able to probe the massive star-formation process as a function of environment and evolutionary stage. Moreover, the sequential behavior of massive star formation can be probed by combining our catalog with the results of Kawamura et al. (2009), who matched NANTEN GMCs with optical SCs younger than 10 Myr (taken from Bica et al. 1996) in order to define Type 3 GMCs. In the following analysis, we use positions as reported by Bica et al. (1996), but caution the reader that the exact central positions for these clusters may not be well known since some of the sources could be extended OB associations. Nonetheless, these young SCs likely represent a more evolved 

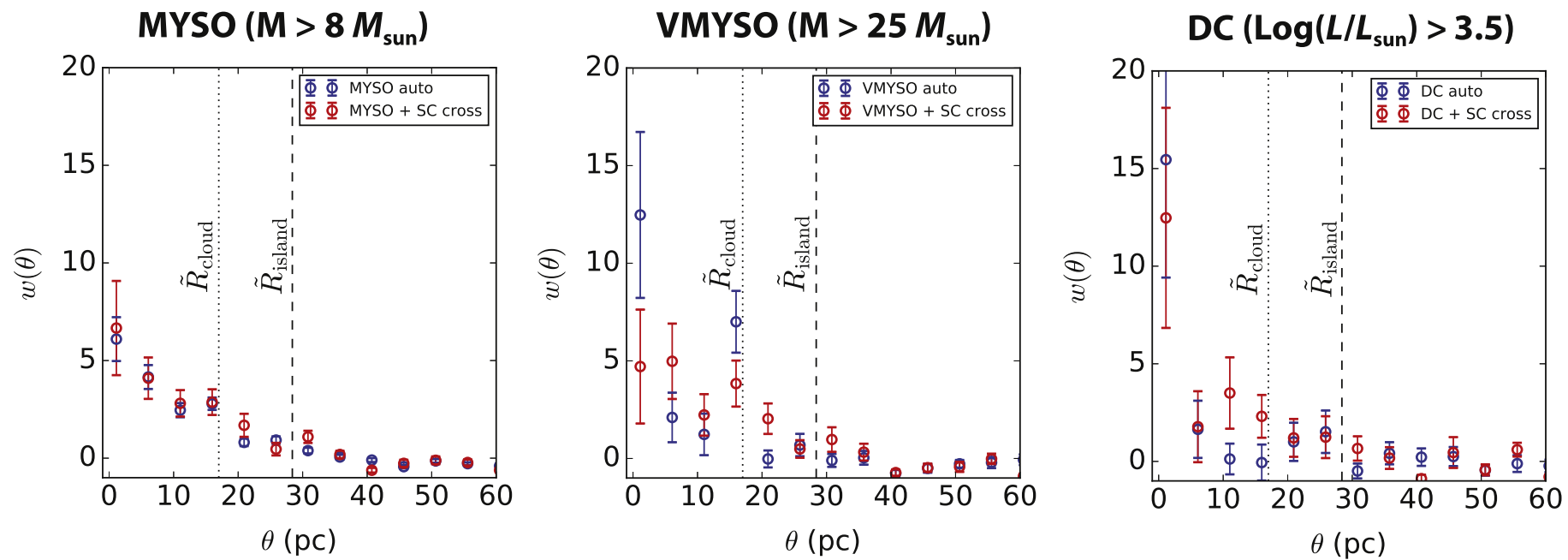

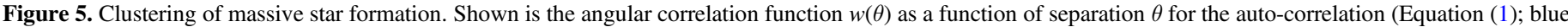
points) and cross-correlation with SCs (Equation (2); red points). The three panels show the results for MYSOs, VMYSOs, and DCs, respectively (see the text).

generation of massive stars compared to the YSOs traced by SAGE and HERITAGE, as they have already emerged from their parent clouds to shine bright at optical wavelengths.

\subsubsection{Angular Correlation Function}

We investigate the clustering of massive star formation by using an angular correlation function. The Landy-Szalay estimator (Landy \& Szalay 1993) calculates the correlation $(w \theta)$ through

$$
w(\theta)=\frac{n_{\mathrm{DD}}(\theta)-2 n_{\mathrm{DR}}(\theta)+n_{\mathrm{RR}}(\theta)}{n_{\mathrm{RR}}(\theta)},
$$

where $n$ represents the number of pair counts between "true" data (subscript D) and "random" data (subscript R) as a function of angular distance $\theta$. Equation (1) computes the intrinsic correlation (or rather: clustering) of a data set, i.e., its "auto-correlation," but it can be generalized for two different data sets (Bradshaw et al. 2011) to compute a "crosscorrelation"

$$
w(\theta)=\frac{n_{\mathrm{D}_{1} \mathrm{D}_{2}}(\theta)-2 n_{\mathrm{D}_{1} \mathrm{R}_{2}}(\theta)-n_{\mathrm{R}_{1} \mathrm{D}_{2}}(\theta)+n_{\mathrm{R}_{1} \mathrm{R}_{2}}(\theta)}{n_{\mathrm{R}_{1} \mathrm{R}_{2}}(\theta)} .
$$

Fundamentally, $(w \theta)$ gives the clustering of a set of points containing positional information in excess over what is expected from a random distribution of points in the same field. Thompson et al. (2012) and Kendrew et al. (2012, 2016) applied Equations (1) and (2) to the distribution of clumps and bubbles drawn from large surveys in the Galactic plane, and demonstrated the use of angular correlation functions to the study of (massive) star formation and its relation to larger-scale structures in the ISM. Here, we apply a similar methodology to the MYSO (569 sources), VMYSO (101 sources), and DC (36 sources) catalogs. We used the public code by S. Kendrew (Kendrew 2015), which makes use of the Astropy package (Astropy Collaboration et al. 2013), and adapted this code for our specific analysis. Random catalogs were constructed over the extent of the LMC $(71 \leqslant$ R.A. $\leqslant 89,-71 \leqslant$ decl. $\leqslant-65)$ with a sample size that is 50 times as large as the input ("true") data catalog to ensure an adequate sampling of the covered area. The uncertainty in $w(\theta)$ is determined using 100 bootstrap resamples (Ling et al. 1986), where pseudo data sets were generated by sampling points with replacement from the ("true") input catalog, while maintaining the same number of sources as the input catalog. The correlation function is then calculated for each of the bootstrap samples: we report the mean and its $1 \sigma$ uncertainty. For the cross-correlations, the pair counts were normalized to account for different catalog sizes. We bin the results in steps of $\Delta \theta=5 \mathrm{pc}$, and start our binning at $1 \mathrm{pc}$ to exclude pair counts of data with themselves. In each panel, we show the auto-correlation of the respective samples, as well as their cross-correlation with the SC sample.

Figure 5 shows the results of this routine. We compare the correlations to the median radius of all islands $\left(\tilde{R}_{\text {island }}=28.4 \mathrm{pc}\right)$ and clouds $\left(\tilde{R}_{\text {cloud }}=17.0 \mathrm{pc}\right)$ identified by the dendrogram algorithm (Section 4), where the radii are estimated assuming spherical symmetry through $R=\sqrt{A / \pi}$, where $A$ represents the surface area of the island/cloud in $\mathrm{pc}^{2}$. Not surprisingly, all correlations show clustering $(w \theta>0)$ at $\theta \leqslant \tilde{R}_{\text {island }}$ and $\theta \leqslant \tilde{R}_{\text {cloud }}$, implying that massive star formation predominantly occurs within the boundaries of an island or cloud. The error bars for the VMYSOs and DCs are relatively large because of the small sample size. Note, however, that the strongest clustering for all sources occurs toward the smallest scales, i.e., $\theta \leqslant 10 \mathrm{pc}$, significantly less than $\tilde{R}_{\text {island }}$ and $\tilde{R}_{\text {cloud }}$. The cross-correlations with SCs show similar trends compared to the respective auto-correlations, suggesting that MYSOs, VMYSOs, and DCs all reside close to SCs. In summary, we conclude that massive star-formation clusters on scales much smaller than the size of parent islands and/or clouds, and that this clustering holds over different generations on timescales of up to $10 \mathrm{Myr}$ (Bica et al. 1996).

\subsubsection{The Presence of SCs and the Rate of Massive Star Formation in GMCs}

An alternative way of quantifying the connection between multiple generations of massive star formation is illustrated in Figure 6. We define a search radius $R_{\mathrm{S}}$ around each SC, along with a distance $d$ between an SC and each peak column density $N_{\max }$. Stellar clusters are then matched with $N_{\max }$ (and their parent cloud) if they fall within the defined value of the search radius, i.e., $d \leqslant R_{\mathrm{s}}$. This routine provides us with a set of $N_{\max }$ 


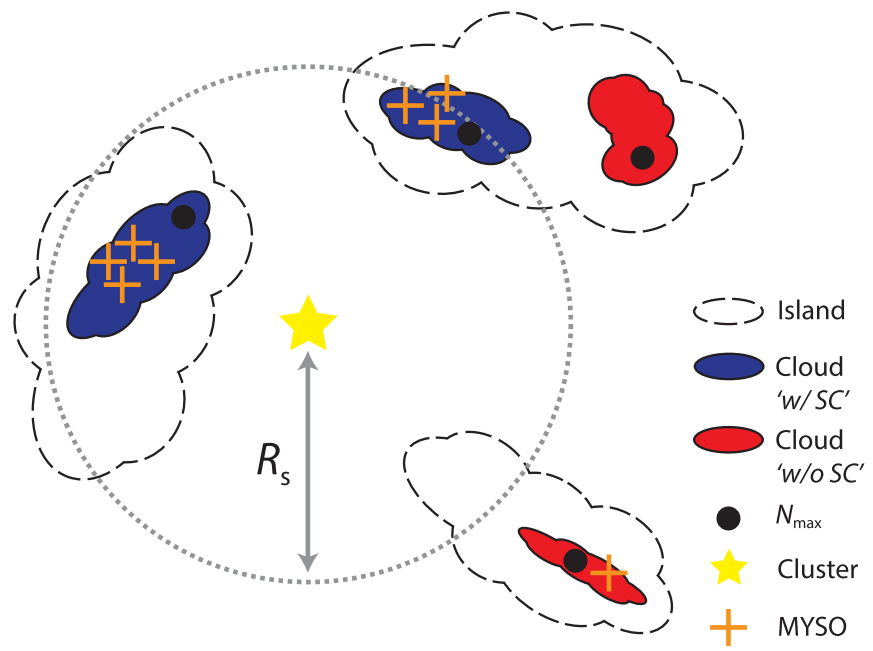

Figure 6. Cartoon depicting the method associating stellar clusters (SCs) with clouds. Our cloud decomposition (Section 4.2) distinguishes between "islands" (long dashed lines), "clouds" (solid lines), and column density peaks $N_{\max }$; see also Figure 2. We define a search radius, $R_{\mathrm{s}}$, around each $\mathrm{SC}$ of our sample. We then separate $N_{\max }$ and their parent clouds that fall within this search radius ("w/SC"; blue clouds), from those that fall outside of the search radius ("w/o SC"; red clouds). We then compare the amount of MYSOs/VMYSOs/DCs found in both samples (Figure 7).

and parent clouds that are located within $R_{\mathrm{S}}$ of SCs (clouds with SCs; "w/SC"), and a set that falls outside of the search radius around SCs (clouds without SCs; "w/o SC"). After this, we count the amount of MYSOs/VMYSOS/DCs associated with each individual cloud. The advantage of this analysis over the use of the angular correlation functions (Equations (1) and (2)) is that we can quantify the connection between MYSOs/ VMYSOs/DCs and SCs, while directly relating this to the parent molecular cloud and its global properties, such as mass $\left(M_{\text {cloud }}\right)$ and average surface density $\left(\left\langle\Sigma_{\text {cloud }}\right\rangle\right)$. To test the significance of our findings, we compare the results with an identical analysis, but using data where MYSO/VMYSO/DCs have been distributed randomly within islands, keeping the total number of sources per island constant.

In the following analysis, we will use search radii of $R_{\mathrm{s}}=10,30$, and $100 \mathrm{pc}$. Note that by using the SC catalog from Kawamura et al. (2009), we limit the analysis to Type 3 GMCs. Indeed, we find a median $\bar{d} \sim 370 \mathrm{pc}, 280 \mathrm{pc}$, and $60 \mathrm{pc}$ for Type 1, 2, and 3 clouds, respectively, confirming the close association of these SCs with Type 3 GMCs.

The results are shown in Figure 7. We note upfront that if the presence of SCs would increase the amount of MYSOs/ VMYSOs/DCs in clouds, we would expect to see a larger object count per cloud in the "w/SC" sample compared to that found with a random distribution of objects. That is, we would observe a "flatter" distribution in Figure 7 compared to the randomizations. Clearly, Figure 7 shows that massive star formation is significantly boosted in clouds found within $10 \mathrm{pc}$ of an SC. The same result is also apparent by comparing the histograms of w/SC and w/o SC samples. At $R_{\mathrm{s}}=10$, we find a clear dichotomy between both samples, where the w/SC sample are shown to contain many more sources than the $\mathrm{w} / \mathrm{o}$ SC sample. This dichotomy is most pronounced for the MYSOs and VMYSOs. More specifically, we find that with $R_{\mathrm{s}}=10, \sim 65 \% / 90 \%$ of the clouds in the w/o SC sample $(d>10 \mathrm{pc})$ are devoid of any MYSO/VMYSOs, whereas this fraction is only $\sim 15 \% / 45 \%$ for the clouds in the $\mathrm{w} / \mathrm{SC}$ sample $(d<10 \mathrm{pc})$. We conclude that clouds within $10 \mathrm{pc}$ of a SC have much higher MYSO/VMYSO (and DC) number counts, implying a correlation between the presence of an SC and an increased rate of massive star formation over the past $\sim 10^{5}$ years.

By increasing our search radius to $R_{\mathrm{s}}=30 \mathrm{pc}$ and $R_{\mathrm{s}}=100 \mathrm{pc}$, the number of clouds in the w/SC sample eventually exceeds that of the w/o SC sample. We find that by increasing $R_{\mathrm{s}}$, the dichotomy in number counts between the $\mathrm{w} / \mathrm{SC}$ and $\mathrm{w} / \mathrm{o}$ SC sample disappears, causing the histograms in Figure 7 to converge. This implies that the correlation between the presence of SCs and the rate of massive star formation becomes less pronounced at larger distances.

Table 2 shows that VMYSOs are almost exclusively found within $30 \mathrm{pc}$ of an SC. A similar trend is seen for DCs, which again may imply that DCs represent a part of the VMYSO population (Section 5.1). Thus, the connection between different generations of massive stars may be stronger for O-type progenitor stars (VMYSOs; Section 5.1) than B-type progenitor stars (MYSOs). Finally, note that $35 \%$ of $N_{\max }$ are not found within $R_{\mathrm{s}}=100 \mathrm{pc}$, even though we established that the median radius of islands and clouds are $28.4 \mathrm{pc}$ and $17.0 \mathrm{pc}$, respectively. This results from the fact that many GMCs are far from spherical (Figure 2) and may be better represented by a filamentary-like morphology.

Figure 7 shows that the amount of MYSOs/VMYSOs may vary greatly between the w/SCs and w/o SCs cloud samples. However, the associated mass $\left(M_{\text {cloud }}\right)$ and average surface density $\left(\left\langle\Sigma_{\text {cloud }}\right\rangle\right)$ distributions of both samples (Figure 7$)$ are remarkably similar. Thus, the rate of massive star formation in clouds near SCs does not appear to correlate strongly with these specific cloud properties.

\section{DISCUSSION}

In this work, we have presented the study of a unique data set that offers a galaxy-wide view of molecular clouds $(M \geqslant$ $\left.3 \times 10^{4} M_{\odot}\right)$, young $\left(\sim 10^{5}\right.$ year $)$ sources on their way to becoming massive stars $\left(M>8 M_{\odot}\right)$, and young $(<10 \mathrm{Myr})$ optical SCs. The sheer size of the data set allowed us to identify the location, clustering, and follow the propagation of massive star formation in GMCs.

In the LMC, massive stars do not typically form at the highest column densities nor center of their parent GMCs at the $\sim 6 \mathrm{pc}$ resolution of our observations (Figures 2 and 3). Half of our sample of MYSOs, VMYSOs, and DCs are formed $\sim 10 \mathrm{pc}$ away from local column density peaks (Figure 4). Massive starformation clusters over different generations and on scales much smaller than the parent molecular cloud (Figure 5), regardless if we include the diffuse parts of the GMCs ("islands") or focus on the highest column density structures alone ("clouds"). While the rate of massive star formation is significantly boosted in clouds near SCs (Figure 7), comparison of molecular clouds associated with SCs with those that are not reveals no significant difference in total mass and average surface density.

\subsection{The Location of Massive Star Formation in GMCs}

The dearth of MYSOs at high column densities in GMCs (Section 5.1) merits further discussion. We have ruled out if completeness systematically affects our analysis (Section 3.1). Alternatively, feedback from massive stars can dynamically 


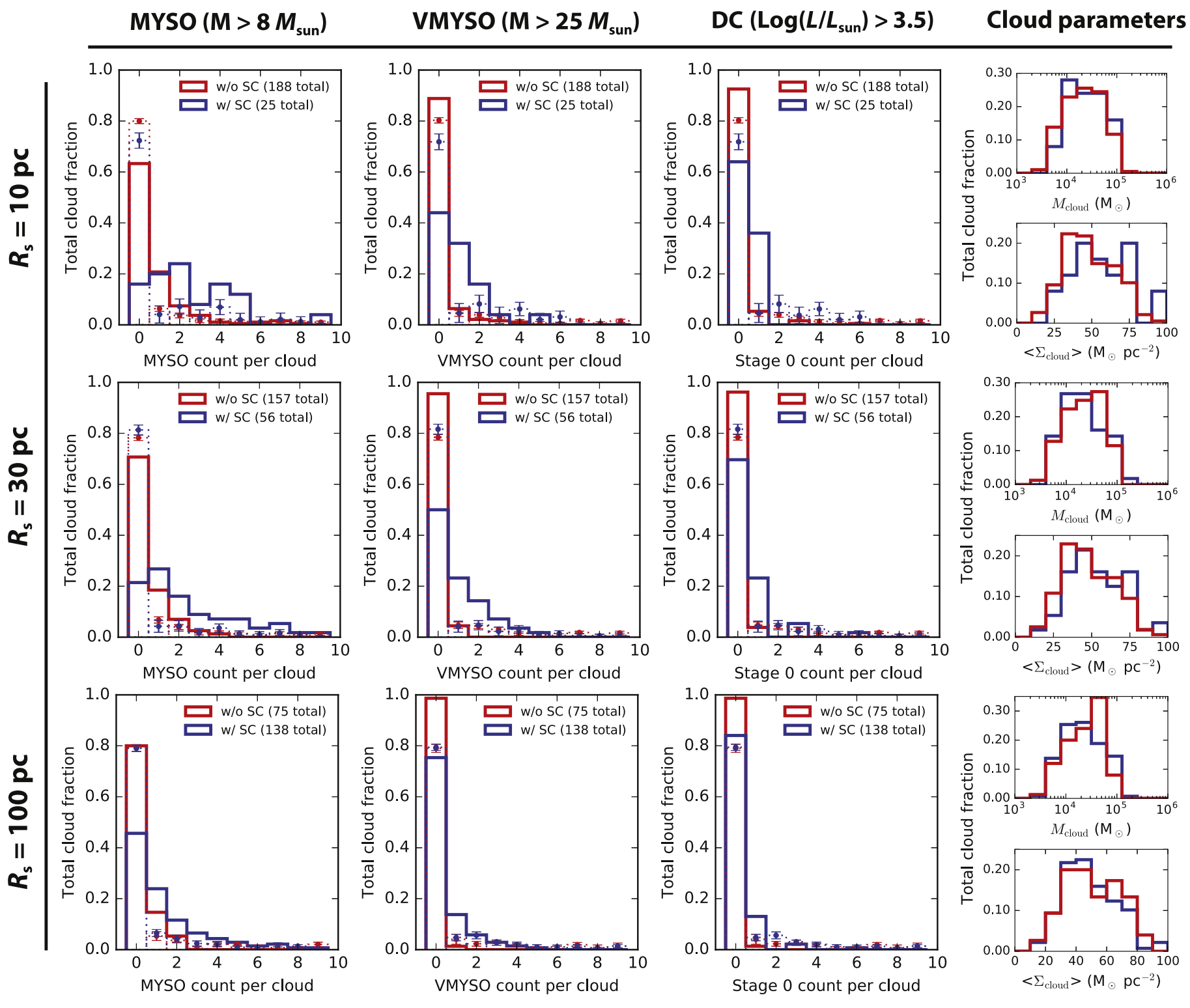

Figure 7. Presence of stellar clusters and the rate of massive star formation in Type 3 clouds. Plotted are results for MYSOs, VMYSOs, and DCs for different search radii $R_{\mathrm{s}}: 10,30$, and $100 \mathrm{pc}$ (see the text and Figure 6). We separate $N_{\max }$ (and their associated parent clouds/islands; Figure 2) at distances of $d \leqslant R_{\mathrm{S}}$ ("w/SC"; (blue solid histogram) from those at $d \geqslant R_{\mathrm{S}}$ ("w/o SC"; red solid histogram), and count the amount of objects (MYSOs, VMYSOs, and DCs, respectively) located within each individual cloud. Histograms are normalized to their respective total number count of clouds (shown in upper right) to compute the total cloud fraction. The results are compared to a situation where the same number of objects (MYSOs, VMYSOs, and DCs) are distributed randomly within islands: the dotted histograms show the mean and $1 \sigma$ uncertainty of 100 randomizations. The far right column show the distributions of mass $M_{\text {cloud }}$ and average surface density $\left\langle\Sigma_{\text {cloud }}\right\rangle$ of the parent clouds.

Table 2

Correlation between SCs and MYSOs/VMYSOs/DCs

\begin{tabular}{lllc}
\hline \hline & $R_{\mathrm{s}}=10 \mathrm{pc}$ & $R_{\mathrm{s}}=30 \mathrm{pc}$ & $R_{\mathrm{s}}=100 \mathrm{pc}$ \\
\hline MYSOs & $31 \%(13 \%)$ & $65 \%(18 \%)$ & $91 \%(51 \%)$ \\
VMYSOs & $39 \%(8 \%)$ & $89 \%(18 \%)$ & $98 \%(52 \%)$ \\
DCs & $26 \%(9 \%)$ & $82 \%(14 \%)$ & $97 \%(47 \%)$ \\
\hline
\end{tabular}

Note. Percentage of MYSOs/VMYSOs/DCs associated with clouds found within search radius $R_{\mathrm{s}}$ from SCs. In parentheses, we provide the same numbers derived through 100 randomizations of the same number of objects (MYSOs, VMYSOs, and DCs) within islands.

alter the cloud material, which may lead to an apparent offset between young massive stars and high column density material. However, given the estimated age of our sample of
YSOs $\left(\sim 10^{5}\right.$ year; Section 3$)$, it is unlikely that we are tracing feedback processes on the physical scales we probe $(\gtrsim 6 \mathrm{pc}$; Section 4.1). In fact, it is unclear when MYSOs start ionizing their surroundings (Churchwell 2002; Hoare \& Franco 2007): current galaxy-wide LMC radio maps of free-free emission (Dickel et al. 2005; Hughes et al. 2007) do not have the angular resolution to asses if some of our MYSO/VMYSO/DC sources have reached the ultra-compact $\mathrm{H}$ II region phase. Even if we assume that our sources have started ionizing their surroundings, analytical solutions (Spitzer 1978; Dyson \& Williams 1980), 1D simulations (Raga et al. 2012), and turbulent 3D simulations (Tremblin et al. 2014) reveal that H II regions in typical molecular cloud conditions only reach sizes of $\lesssim 0.5 \mathrm{pc}$ within $10^{5}$ year, which is small compared to the resolution of our column density maps $(\sim 6 \mathrm{pc})$. Thus, the 
timescales involved are incompatible with our young massive stars having created several parsec-sized cavities within their natal clouds. Indeed, high-resolution $\mathrm{H} \alpha$ imaging (Figure 2) do not show the indications of large-scale feedback processes, confirming the embedded nature of our MYSO sample.

As a caveat, we note that the presence of internal heating sources can elevate the local dust temperature, overestimating the mass-averaged temperature along the line of sight, and thereby underestimating the total observed column density. Conversely, in the absence of an internal heating source, the observed SED will be biased toward the irradiated outskirts of clouds or cores as opposed to the dark, cold cores containing the bulk of the mass. These effects are inherent to FIR SED fitting, and have been addressed in many studies (e.g., Malinen et al. 2011; Ysard et al. 2012). The underestimation of mass appears to be larger for starless than for protostellar cores (Malinen et al. 2011), because internal heating renders dust more easily visible and estimations of cloud masses become more reliable. As noted by Juvela et al. (2013), quantifying the extent to which our column densities are affected by the absence/presence of, e.g., YSOs could only be alleviated by knowledge of the temperature structure of the source and the detailed structure of the molecular cloud (i.e., density and lineof-sight depth). Alternatively, we can resort to gas-based column density tracers: the MAGMA CO data show very similar distributions (Figure 2); however, we have already pointed out that ${ }^{12} \mathrm{CO}(1-0)$ has observational limitations on its own (Section 4). Future observations of GMCs in various tracers of different critical densities will allow us to map the internal structure of GMCs at a high dynamical range. In this way, we will be able to quantify whether our FIR-derived column density maps are significantly affected by line-of-sight temperature gradients, and how this impacts our results on the location of MYSOs in GMCs (Section 5.1).

\subsubsection{Comparison with Galactic Studies}

Galactic studies of (massive) star formation are traditionally complicated by confusion, large angular scales, and distance ambiguity. Our study targeting massive star formation in the LMC circumvents these limitations and, as a result, there are (as of yet) no Milky Way studies of similar size, combining observations of hundreds of GMCs, associated SCs, and a complete census of embedded massive star formation over the past $\sim 10^{5}$ year, which allows for a statistical study of massive star formation and its dependence on environment and evolutionary state.

In the Galaxy, massive stars may form within IRDCs (e.g., Beuther et al. 2005; Rathborne et al. 2006). One might question if the HERITAGE maps offer sufficient spatial resolution to be sensitive to typical IRDCs and star-forming clumps such as those seen in (nearby) Galactic clouds. Surely, on small scales, the column densities at the position of the MYSOs may be very high, and beam dilution may render these sites undetectable to our observations. IRDCs have typical sizes of $\sim 5 \mathrm{pc}$ (Simon et al. 2006), while massive star forming clumps have sizes of order $\sim 1$ pc (Tan et al. 2014, p. 149). Both of these structures would be unresolved at the resolution of our $N\left(\mathrm{H}_{2}\right)$ map $(\sim 6 \mathrm{pc}$; Section 4). In this respect, a proper exercise is to consider if we would be able to detect the nearest example of massive star formation, located within the Orion A molecular cloud. Orion A has a surface area of $\sim 2200 \mathrm{pc}^{2}$ and contains $\sim 10^{5} M_{\odot}$ of molecular mass (Wilson et al. 2005), large and massive enough to be resolved and detected in the HERITAGE maps. On the northern tip of the cloud lies the "integral-shaped filament" (ISF; Bally et al. 1987), a dense ridge $(9 \times 0.5 \mathrm{pc})$ containing $\sim 5 \times 10^{3} M_{\odot}$ of molecular gas (Bally et al. 1987; Berné et al. 2014). While the ISF only comprises $1 / 500$ of the surface area of Orion A (Wilson et al. 2005), it contains 1/20 of its mass, thereby locally increasing the column density by a factor of $\sim 25$. If we assume that Orion A (cloud) and the ISF (filament) are hierarchically perched on top of one another (Wilson et al. 2005), we can estimate the observed column density contrast of the Orion ISF region with respect to the entire Orion A cloud, taking into account the beam-filling factor $f$ of the ISF in our $6 \mathrm{pc}$ resolution maps $\left(f_{\mathrm{ISF}} \sim 0.1\right)$. We write the column density contrast as $\eta=\left(N_{\mathrm{H}_{2}}\right.$ [Ori A] + $\left.f_{\text {ISF }} N_{\mathrm{H}_{2}}[\mathrm{ISF}]\right) /\left(N_{\mathrm{H}_{2}}\right.$ [Ori A] $) \sim 3.5$. We conclude that the ISF, and thereby a site of massive star formation like Orion, should be detectable in our column density maps of the LMC.

The above derivation illustrates a key point: massive stars in the LMC do not appear to form in environments with masses similar to that of Galactic IRDCs $\left(\sim 5 \times 10^{3} M_{\odot}\right.$; Simon et al. 2006). Still, we would expect massive stars to form in density enhancements unresolved at our $\sim 6 \mathrm{pc}$ resolution, consistent with gas clumps and massive cores observed in the Galaxy ( $\lesssim 1$ pc; Tan et al. 2014, p. 149). Our results illustrate that the clumps and cores forming massive stars are created outside of the densest, most opaque regions of GMCs (Figures 2-4). To estimate an upper limit for the mass of these systems, we use the median surface density of all GMCs in the LMC, equalling 23 and $37 M_{\odot} \mathrm{pc}^{-2}$ for islands and clouds, respectively. At these surface densities and assuming a Gaussian beam with FWHM $=6 \mathrm{pc}$, a compact (unresolved) gas clump of mass $M_{\mathrm{cl}}=500 M_{\odot}$, massive enough to form a cluster of mass $M_{\star}$ containing a maximum stellar mass of $\sim 25 M_{\odot}$, would lead to a column density contrast $\eta \sim 1.5$ (assuming an efficiency of $M_{\mathrm{cl}} / M_{\star} \sim 0.5$ and a Kroupa initial mass function; Kroupa 2001; Tan et al. 2014, p. 149). Such a column density contrast may be confused with the background GMC. Alternatively, on the scales of individual gas clumps and massive cores $(\lesssim 1 \mathrm{pc})$, the MYSOs/VMYSOs/DCs may simply have destroyed their natal star-forming clump, given that the current estimated destruction timescale $\left(\sim 3 \times 10^{5}\right.$ year; Seale et al. 2012) is of the order of the estimated age of our MYSO sample ( $\sim 10^{5}$ year; Section 3$)$.

We conclude that massive stars in the LMC appear to form in clumps with masses of up to $\lesssim 500 M_{\odot}$, which is consistent with current theories and observations of massive star formation (see Tan et al. 2014 p. 149, for a recent comprehensive review). However, the observation that massive stars (and, presumably, their natal clumps) form outside of the main body of molecular gas in GMCs is puzzling, and may provide important clues to the collapse of molecular clouds and the initial conditions that may lead to the formation of massive stars (see Section 6.3). We note that these results may also apply to massive star formation in the Galaxy, given that most Galactic IRDCs show no sign of active star formation (Chambers et al. 2009), while the recently discovered "giant molecular filaments" in the Galaxy (Jackson et al. 2010; Ragan et al. 2014) reveal many massive cores and ultra-compact H II regions around the edges of the giant filaments (Abreu-Vicente et al. 2016).

We argue that in order to advance our understanding of the location and formation of massive stars within GMCs, it is 
essential to consider entire GMC complexes (including the potential influence of external factors; Section 6.3), instead of merely focusing on "hot spots" that appear to be prime candidates for the formation of massive stars (i.e., IRDCs). In this regard, high-resolution follow-up observations of GMCs complexes with the Atacama Large Millimeter Array (e.g., Indebetouw et al. 2013; Fukui et al. 2015; Nayak et al. 2016) together with sensitive observations of MYSOs with the James Webb Space Telescope will provide suitable tools to advance our understanding on the location, clustering, and propagation of massive star formation and its relation to the large-scale structure of GMCs and the ISM of galaxies.

\subsection{The Clustering and Propagation of Massive Star Formation in GMCs}

Figure 7 revealed that there is a strong dichotomy in the massive star-formation rate between Type 3 clouds, depending on their location in regards to SCs. Specifically, massive star formation is significantly boosted in clouds near SCs, with the effect becoming less pronounced at larger distances from SCs (Figure 7). The results suggest a connection between different generations of massive stars on timescales of up to $10 \mathrm{Myr}$. It is tempting to take this result as evidence for triggered star formation, where, once star formation is initiated, the interaction of the newly formed massive stars with their environment drives the formation of the next generation (see Section 6.3).

One may argue that massive star formation can exclusively be found in regions with certain physical conditions (e.g., above a mass or density threshold) and that it is only natural to find massive stars clustered in these particular regions. After all, it is very well known that massive stars form almost exclusively in clustered environments (Lada \& Lada 2003). However, the rate of massive star formation in clouds near SCs does not appear to correlate with the global properties $M_{\text {cloud }}$ and $\left\langle\Sigma_{\text {cloud }}\right\rangle$ (Figure 7). This may be related to the results from Section 5: massive star formation takes place on scales much smaller than islands (Table 1) as well as clouds (Figure 5). These results indicate that massive star formation is a local process within GMCs. Massive star formation as a local process would disconnect the rate of massive star formation from the global cloud properties $M_{\text {cloud }}$ and $\left\langle\Sigma_{\text {cloud }}\right\rangle$. We note that similar results were obtained for nearby molecular clouds (Heiderman et al. 2010; Lada et al. 2010), where star formation appears poorly correlated with total molecular cloud mass, but is instead closely related to the dense gas fraction within molecular clouds. However, it is important to note that these results were based on low-mass star formation: it is unclear if the same laws apply to high-mass star formation (Section 6.3). Unfortunately, our observations do not have the resolution to discern between dense and diffuse gas within the GMCs: higher-resolution data resolving the intrinsic GMC structure is needed to discern if low-mass and high-mass stars form in the same way, or if they form through different pathways.

\subsection{The Modes of Massive Star Formation and Comparison with Earlier Works}

At this point, we reiterate the two main results presented in this work. First, MYSOs do not form at column density peaks of GMCs. Second, massive star formation is more active in clouds close to young SCs. As noted in Section 6.1.1, these results may provide important clues to the collapse of molecular clouds and the initial conditions that lead to the formation of massive stars. Below, we explore routes to the formation of massive stars identified in the literature that may be consistent with our observations.

Numerical and analytical studies have identified mechanisms that can lead to the formation of massive cores on the edges of molecular clouds (Burkert \& Hartmann 2004; Heitsch et al. 2008; Pon et al. 2011; Li et al. 2016). For example, "edge effects" arise in collapsing finite cloud sheets, where material accumulates and fragments at the outer boundaries of the cloud where the gravitational acceleration is greatest (Burkert \& Hartmann 2004). In addition, Heitsch et al. (2008) studied the formation of molecular clouds in largescale colliding flows, and showed that while global collapse of a molecular cloud creates centrally located large-scale filaments, local gravitational collapse can lead to high-mass cores far away from the centers of molecular clouds on timescales much shorter than the global dynamical collapse time.

The above described mechanisms can lead to the "spontaneous" formation of massive stars at the outskirts of molecular clouds. A different train of thought involves the notion that high-mass star formation has to be induced or "triggered" as opposed to their lower-mass counterparts (Shu et al. 1987). The actual driving agents of triggering may vary and act on a wide range of different scales, from galaxy mergers (Woods et al. 2006), galaxy-scale turbulence (Mac Low \& Klessen 2004), spiral arm passages (Roberts 1969), supershells (Tenorio-Tagle \& Bodenheimer 1988), cloud-cloud collisions (Scoville et al. 1986; Fukui et al. 2015), to that of single stars or clusters through "cloud-crushing" (Bertoldi 1989), or the "collect-and-collapse" process (Elmegreen \& Lada 1977; Zavagno et al. 2007). While studies of individual, isolated regions such as RCW 120 (e.g., Zavagno et al. 2010) have unambiguously demonstrated the importance of triggered star formation, its relevance on a larger scale has remained controversial (Dale et al. 2015). The controversy arises largely because most regions of massive star formation show lots of star formation related activity in different stages of evolution, and that makes pinpointing the effects of triggered star formation difficult.

Local gravitational collapse of GMCs as a mode of massive star formation may explain the distribution of young massive stars in the LMC (Figure 3), as this would not a priori favor the central (i.e., highest column density) regions within GMCs as the principle formation site of massive stars. Alternatively, feedback from massive stars may trigger the formation of a next generation in regions of a GMC that may not necessarily correlate with total column density. Both of these scenarios can occur within localized (small) regions of GMCs, consistent with the scale size of clustering versus that of the size of GMCs (Figure 5), and the rate of massive star formation disconnected to the global properties of GMCs (Figure 7). Moreover, triggering as a key mode for massive star formation links different generations of massive stars and would explain the strong correlation between the presence of SCs and the rate of massive star formation (Figure 7). While the importance of induced star formation has been a subject of debate for decades (Shu et al. 1987; Elmegreen 1998; Dale et al. 2015), it appears consistent with results on individual (nearby) massive starforming regions in the Galaxy (Blaauw 1964; Elmegreen \& 
Lada 1977; Povich et al. 2009; Zavagno et al. 2010), and we argue that the close association of exposed clusters with nearby embedded massive stars provides further support for the importance of triggered star formation on a galaxy-wide scale.

\section{CONCLUSIONS}

We have studied massive star formation in GMCs of the LMC using an unbiased sample of $\sim 600$ MYSOs, 200 GMCs, and $\sim 100$ SCs. Unhindered by confusion or luminosity uncertainties that typically hamper Galactic studies, we were able to study the location, clustering, and propagation of massive star formation within GMCs. Our main results are as follows.

1. Our MYSO catalog is complete for Stage 1 MYSOs of mass $M>8 \quad M_{\odot}$, provided that they have mid-IR counterparts (Section 3.1).

2. We find ongoing massive star formation (i.e., over the past $\sim 10^{5}$ year) in $33 \%$ or $48 \%$ of the LMC GMCs, depending on whether we consider "clouds" or "islands" (Section 4.2). We substantiate the classification scheme from Kawamura et al. (2009) by revealing that Type 1 GMCs are (mostly) devoid of massive star formation (Table 1).

3. We find that massive stars do not form at the peak column densities within GMCs at the $\sim 6 \mathrm{pc}$ resolution of our observations (Figures 2 and 3). Specifically, half of our sample of MYSOs/VMYSOs/DCs are located $>10 \mathrm{pc}$ from $N_{\max }$ (Figure 4). We have excluded completeness or feedback as a cause for this result (Section 5).

4. By means of angular correlation functions (Equations (1) and (2); Figure 5), we have demonstrated that MYSOs/ VMYSOs/DCs are strongly clustered on scales much smaller than the size of CO islands and clouds. The autocorrelations show very similar results compared to their respective cross-correlations with SCs, indicating that massive star formation is clustered over different generations on timescales of up to $10 \mathrm{Myr}$.

5. We find that the rate of massive star formation is significantly elevated in clouds near SCs (Figure 7). At the same time, the rate of massive star formation in these clouds appears unrelated to the global cloud properties $M_{\text {cloud }}$ and $\left\langle\Sigma_{\text {cloud }}\right\rangle$. The relative increase in massive star formation becomes less pronounced at larger distances from the SCs.

We argue that massive star formation is a local process within GMCs. It appears that the initial conditions leading to massive star formation do not necessarily occur in the densest, most opaque regions of GMCs. Our results reveal a close connection between different generations of massive stars on timescales of up to $10 \mathrm{Myr}$, which may provide further support for triggering as a key mode for massive star formation, which in its turn could proceed very differently compared to their lower-mass counterparts.

The authors wish to thank Kirill Tchernyshyov and Karl Gordon for many useful discussions, and Henrik Beuther and Sarah Kendrew for comments on an (early) draft of this paper. We thank the MAGMA team for permission to use the DR3 products in advance of publication. B.O. is supported through NASA ADAP grant NNX15AF17G. In addition, this work has greatly benefited from a detailed and helpful report provided by an anonymous referee, which improved both the quality and clarity of this paper.

\section{REFERENCES}

Abreu-Vicente, J., Ragan, S., Kainulainen, J., et al. 2016, A\&A, 590, A131 André, P., Di Francesco, J., Ward-Thompson, D., et al. 2014, in Protostars and Planets VI, ed. H. Beuther et al. (Tucson, AZ: Arizona Univ. Press), 27

André, P., Men'shchikov, A., Bontemps, S., et al. 2010, A\&A, 518, L102

Astropy Collaboration, Robitaille, T. P., Tollerud, E. J., et al. 2013, A\&A, 558, A33

Bally, J., Langer, W. D., Stark, A. A., \& Wilson, R. W. 1987, ApJL, 312, L45 Bernard, J.-P., Reach, W. T., Paradis, D., et al. 2008, AJ, 136, 919

Berné, O., Marcelino, N., \& Cernicharo, J. 2014, ApJ, 795, 13

Bertoldi, F. 1989, ApJ, 346, 735

Beuther, H., Sridharan, T. K., \& Saito, M. 2005, ApJL, 634, L185

Bica, E., Claria, J. J., Dottori, H., Santos, J. F. C., Jr., \& Piatti, A. E. 1996, ApJS, 102, 57

Blaauw, A. 1964, ARA\&A, 2, 213

Bolatto, A. D., Wolfire, M., \& Leroy, A. K. 2013, ARA\&A, 51, 207

Bradshaw, E. J., Almaini, O., Hartley, W. G., et al. 2011, MNRAS, 415, 2626

Burkert, A., \& Hartmann, L. 2004, ApJ, 616, 288

Chambers, E. T., Jackson, J. M., Rathborne, J. M., \& Simon, R. 2009, ApJS, 181,360

Churchwell, E. 2002, ARA\&A, 40, 27

Dale, J. E., Haworth, T. J., \& Bressert, E. 2015, MNRAS, 450, 1199

Dickel, J. R., McIntyre, V. J., Gruendl, R. A., \& Milne, D. K. 2005, AJ, 129,790

Dunham, M. M., Stutz, A. M., Allen, L. E., et al. 2014, in Protostars and Planets VI, ed. H. Beuther et al. (Tucson, AZ: Arizona Univ. Press), 195

Dupac, X., Bernard, J.-P., Boudet, N., et al. 2003, A\&A, 404, L11

Dyson, J. E., \& Williams, D. A. 1980, Physics of the Interstellar Medium (New York: Halsted Press)

Elmegreen, B. G. 1998, in ASP Conf. Ser. 148, Origins, ed. C. E. Woodward, J. M. Shull, \& H. A. Thronson, Jr. (San Francisco, CA: ASP), 150

Elmegreen, B. G., \& Lada, C. J. 1977, ApJ, 214, 725

Evans, N. J., II, Dunham, M. M., Jørgensen, J. K., et al. 2009, ApJS, 181, 321

Fukui, Y., Harada, R., Tokuda, K., et al. 2015, ApJL, 807, L4

Fukui, Y., Kawamura, A., Minamidani, T., et al. 2008, ApJS, 178, 56

Goodman, A. A., Pineda, J. E., \& Schnee, S. L. 2009, ApJ, 692, 91

Gordon, K. D., Roman-Duval, J., Bot, C., et al. 2014, ApJ, 797, 85

Gruendl, R. A., \& Chu, Y.-H. 2009, ApJS, 184, 172

Heiderman, A., Evans, N. J., II, Allen, L. E., Huard, T., \& Heyer, M. 2010, ApJ, 723, 1019

Heitsch, F., Hartmann, L. W., Slyz, A. D., Devriendt, J. E. G., \& Burkert, A. 2008, ApJ, 674, 316

Hoare, M. G., \& Franco, J. 2007, ASSP, 1, 61

Hughes, A., Meidt, S. E., Colombo, D., et al. 2013, ApJ, 779, 46

Hughes, A., Staveley-Smith, L., Kim, S., Wolleben, M., \& Filipović, M. 2007, MNRAS, 382, 543

Indebetouw, R., Brogan, C., Chen, C.-H. R., et al. 2013, ApJ, 774, 73

Jackson, J. M., Finn, S. C., Chambers, E. T., Rathborne, J. M., \& Simon, R. 2010, ApJL, 719, L185

Jameson, K. E., Bolatto, A. D., Leroy, A. K., et al. 2016, ApJ, 825, 12

Juvela, M., Malinen, J., \& Lunttila, T. 2013, A\&A, 553, A113

Kawamura, A., Mizuno, Y., Minamidani, T., et al. 2009, ApJS, 184, 1

Kendrew, S. 2015, milkywayproject_triggering: Correlation functions for two catalog datasets, Astrophysics Source Code Library, record ascl.soft11012K

Kendrew, S., Beuther, H., Simpson, R., et al. 2016, ApJ, 825, 142

Kendrew, S., Simpson, R., Bressert, E., et al. 2012, ApJ, 755, 71

Kenyon, S. J., Hartmann, L. W., Strom, K. M., \& Strom, S. E. 1990, AJ, 99, 869

Könyves, V., André, P., Men'shchikov, A., et al. 2010, A\&A, 518, L106

Kroupa, P. 2001, MNRAS, 322, 231

Lada, C. J., \& Lada, E. A. 2003, ARA\&A, 41, 57

Lada, C. J., Lombardi, M., \& Alves, J. F. 2010, ApJ, 724, 687

Landy, S. D., \& Szalay, A. S. 1993, ApJ, 412, 64

Li, G.-X., Burkert, A., Megeath, T., \& Wyrowski, F. 2016, arXiv:1603.05720

Ling, E. N., Barrow, J. D., \& Frenk, C. S. 1986, MNRAS, 223, 21

Mac Low, M.-M., \& Klessen, R. S. 2004, RvMP, 76, 125

Madden, S. C., Poglitsch, A., Geis, N., Stacey, G. J., \& Townes, C. H. 1997, ApJ, 483, 200

Malinen, J., Juvela, M., Collins, D. C., Lunttila, T., \& Padoan, P. 2011, A\&A, 530, A101 
Meixner, M., Gordon, K. D., Indebetouw, R., et al. 2006, AJ, 132, 2268

Meixner, M., Panuzzo, P., Roman-Duval, J., et al. 2013, AJ, 146, 62

Motte, F., Bontemps, S., Schilke, P., et al. 2007, A\&A, 476, 1243

Mottram, J. C., Hoare, M. G., Davies, B., et al. 2011, ApJL, 730, L33

Nayak, O., Meixner, M., Indebetouw, R., et al. 2016, arXiv:1608.05451

Pietrzyński, G., Graczyk, D., Gieren, W., et al. 2013, Natur, 495, 76

Pineda, J. E., Rosolowsky, E. W., \& Goodman, A. A. 2009, ApJL, 699, L134

Pon, A., Johnstone, D., \& Heitsch, F. 2011, ApJ, 740, 88

Povich, M. S., Churchwell, E., Bieging, J. H., et al. 2009, ApJ, 696, 1278

Raga, A. C., Cantó, J., \& Rodríguez, L. F. 2012, MNRAS, 419, L39

Ragan, S., Henning, T., Krause, O., et al. 2012, A\&A, 547, A49

Ragan, S. E., Henning, T., Tackenberg, J., et al. 2014, A\&A, 568, A73

Rathborne, J. M., Jackson, J. M., \& Simon, R. 2006, ApJ, 641, 389

Roberts, W. W. 1969, ApJ, 158, 123

Robitaille, T. P. 2008, in ASP Conf. Ser. 387, Massive Star Formation: Observations Confront Theory, ed. H. Beuther, H. Linz, \& T. Henning (San Francisco, CA: ASP), 290

Robitaille, T. P., Whitney, B. A., Indebetouw, R., Wood, K., \& Denzmore, P. 2006, ApJS, 167, 256

Roman-Duval, J., Gordon, K. D., Meixner, M., et al. 2014, ApJ, 797, 86

Rosolowsky, E. W., Pineda, J. E., Kauffmann, J., \& Goodman, A. A. 2008, ApJ, 679, 1338

Schneider, N., Csengeri, T., Hennemann, M., et al. 2012, A\&A, 540, L11

Scoville, N. Z., Sanders, D. B., \& Clemens, D. P. 1986, ApJL, 310, L77

Scoville, N. Z., Yun, M. S., Sanders, D. B., Clemens, D. P., \& Waller, W. H. 1987, ApJS, 63, 821

Seale, J. P., Looney, L. W., Wong, T., et al. 2012, ApJ, 751, 42
Seale, J. P., Meixner, M., Sewiło, M., et al. 2014, AJ, 148, 124

Shetty, R., Kauffmann, J., Schnee, S., \& Goodman, A. A. 2009, ApJ, 696, 676

Shu, F. H., Adams, F. C., \& Lizano, S. 1987, ARA\&A, 25, 23

Simon, R., Rathborne, J. M., Shah, R. Y., Jackson, J. M., \& Chambers, E. T. 2006, ApJ, 653, 1325

Smith, R. C. \& MCELS Team 1998, PASA, 15, 163

Spitzer, L. 1978, Physical Processes in the Interstellar Medium (New York: Wiley-Interscience)

Svoboda, B. E., Shirley, Y. L., Battersby, C., et al. 2016, ApJ, 822, 59

Tackenberg, J., Beuther, H., Henning, T., et al. 2012, A\&A, 540, A113

Tan, J. C., Beltrán, M. T., Caselli, P., et al. 2014, in Protostars and Planets VI, ed. H. Beuther et al. (Tucson, AZ: Arizona Univ. Press), 149

Tenorio-Tagle, G., \& Bodenheimer, P. 1988, ARA\&A, 26, 145

Thompson, M. A., Urquhart, J. S., Moore, T. J. T., \& Morgan, L. K. 2012, MNRAS, 421, 408

Tout, C. A., Pols, O. R., Eggleton, P. P., \& Han, Z. 1996, MNRAS, 281,257

Tremblin, P., Anderson, L. D., Didelon, P., et al. 2014, A\&A, 568, A4

Whitney, B. A., Sewilo, M., Indebetouw, R., et al. 2008, AJ, 136, 18

Williams, J. P., de Geus, E. J., \& Blitz, L. 1994, ApJ, 428, 693

Wilson, B. A., Dame, T. M., Masheder, M. R. W., \& Thaddeus, P. 2005, A\&A, 430,523

Wong, T., Hughes, A., Ott, J., et al. 2011, ApJS, 197, 16

Woods, D. F., Geller, M. J., \& Barton, E. J. 2006, AJ, 132, 197

Ysard, N., Juvela, M., Demyk, K., et al. 2012, A\&A, 542, A21

Zavagno, A., Pomarès, M., Deharveng, L., et al. 2007, A\&A, 472, 835

Zavagno, A., Russeil, D., Motte, F., et al. 2010, A\&A, 518, L81 